\title{
A bootstrapped spectral test for adequacy in weak ARMA models
}

\author{
BY Ke ZHU \\ Institute of Applied Mathematics, Chinese Academy of Sciences, Haidian District, \\ Zhongguancun, Beijing, China \\ kzhu@amss.ac.cn \\ AND WAI KEUNG Li \\ Department of Statistics and Actuarial Science, University of Hong Kong, Pokfulam Road, \\ Hong Kong \\ hrntlwk@hku.hk
}

\section{SUMMARY}

This paper proposes a Cramér-von Mises (CM) test statistic to check the adequacy of weak ARMA models. Without posing a martingale difference assumption on the error terms, the asymptotic null distribution of the CM test is obtained. Moreover, this CM test is consistent, and has nontrivial power against the local alternative of order $n^{-1 / 2}$. Due to the unknown dependence of error terms and the estimation effects, a new block-wise random weighting method is constructed to bootstrap the critical values of the test statistic. The new method is easy to implement and its validity is justified. The theory is illustrated by a small simulation study and an application to S\&P 500 stock index.

Some key words: Block-wise random weighting method; Diagnostic checking; Least squares estimation; Spectral test; Weak ARMA models; Wild bootstrap.

JEL classifications: C01; C12; C22.

\section{INTRODUCTION}

After the seminal work of Box and Pierce (1970) and Ljung and Box (1978), diagnostic checking has been an important step in the application of the following $\operatorname{ARMA}(p, q)$ model:

$$
y_{t}=\sum_{i=1}^{p} \phi_{i} y_{t-i}+\sum_{i=1}^{q} \varphi_{i} \varepsilon_{t-i}+\varepsilon_{t},
$$

where $\varepsilon_{t}$ are error terms with mean zero. As usual, we say that model (1) is weak when $\left\{\varepsilon_{t}\right\}$ is an uncorrelated sequence, and that model (1) is strong when $\left\{\varepsilon_{t}\right\}$ is an iid sequence; see, e.g., Francq and Zakoïan (1998). Up to now, the most famous diagnostic checking tools for model (1) are the portmanteau tests in Box and Pierce (1970) and Ljung and Box (1978). However, their asymptotic null distributions are only valid for strong ARMA models; see, e.g., Romano and Thombs (1996) and Francq, Roy, and Zakoïan (2005). Moreover, empirical studies in Franses and Van Dijk (1996) and Tsay (2005) demonstrated that many economic and financial series 
follow an ARMA model with uncorrelated errors (e.g., ARCH-type errors). In addition, Francq (2005) indicated that many nonlinear models admit a weak ARMA representation. Thus, it is meaningful to consider diagnostic checking for weak ARMA models.

Based on either observable series (i.e., $p=q=0$ ) or residual series, a huge literature so far has been focused on testing model adequacy in weak ARMA models. These existing tests are roughly categorized into two types: time domain correlation-based tests and frequency domain periodogram-based tests. The tests in the first category usually use the autocorrelations up to lag $m$ (a user-chosen integer), so they are unable to detect serial correlations beyond lag $m$; see, e.g., Romano and Thombs (1996), Lobato (2001), and Horowitz, Lobato, Nankervis, and Savin (2006) for observable series, or Francq, Roy, and Zakoïan (2005) and Delgado and Velasco (2011) for residual series. To avoid selecting $m$, Escanciano and Lobato (2009) and Escanciano, Lobato, and Zhu (2013) derived a data-driven portmanteau test under the assumption that $\varepsilon_{t}$ is a martingale difference sequence (MDS). However, it is unclear whether their tests are applicable if $\varepsilon_{t}$ is not an MDS.

Since the correlation-based tests are inconsistent, the periodogram-based tests in the second category have drawn more attention in the literature; see, e.g., Durlauf (1991) and Deo (2000) for earlier works. Under the assumption that $\varepsilon_{t}$ admits a linear process of iid innovations, the smoothing rescaled periodogram test in Paparoditis (2000, 2001) and generalised likelihood ratio test in Fan and Zhang (2004) can be used to check the whiteness of residual series; see, e.g., Fan and Jiang (2007) for more works on smoothed tests. However, the two aforementioned smoothed models). Under the assumption that $\varepsilon_{t}$ is an MDS, many spectral tests have been constructed by Delgado, Hidalgo, and Velasco (2005), Escanciano (2006, 2007), and Escanciano and Velasco (2006). Recently, Shao (2011a) proposed a spectral test for observable series without the MDS assumption on the error terms. A natural but important extension is to construct spectral tests 60 for residual series when $\varepsilon_{t}$ is not an MDS. Under the assumption that $\varepsilon_{t}$ is $\operatorname{GMC}(8)$ (a condition weaker than MDS), Shao (2011b) proved the validation of the kernel-based spectral test in Hong (1996), where the definition of GMC is given in Remark 3 below, and the lag $m$ as a bandwidth grows slowly with the sample size.

This paper proposes a Cramér-von Mises (CM) spectral test statistic to check the adequacy of 65 weak ARMA models. Under certain conditions allowing for non-MDS error terms, the asymptotic null distribution of the CM test is obtained. Moreover, this CM test is consistent, and has nontrivial power against local alternatives of order $n^{-1 / 2}$. Due to the unknown dependence structure of error terms and the estimation effects, our null distribution is no longer asymptotically pivotal. This is also the main challenge for other spectral tests in weak ARMA models. To overto come it, a new block-wise random weighting (BRW) method is constructed to bootstrap critical values of the CM test. The new method is easy to implement and its validity is justified. The theory is illustrated by a small simulation study and an application to S\&P 500 stock index.

This paper is organized as follows. Section 2 gives our test statistic and establishes its asymptotic theory. Section 3 proposes a BRW method and proves its validation. Simulation results are reported in Section 4. A real example is provided in Section 5. Concluding remarks are offered in Section 6. All of the proofs are given in the Appendix. Throughout the paper, $A^{\prime}$ is the transpose of matrix $A,|A|=\left(\operatorname{tr}\left(A^{\prime} A\right)\right)^{1 / 2}$ is the Euclidean norm of a matrix $A,\|A\|_{s}=\left(E|A|^{s}\right)^{1 / s}$ is the $L^{s}$-norm $(s \geq 1)$ of a random matrix, $o_{p}(1)\left(O_{p}(1)\right)$ denotes a sequence of random numbers converging to zero (bounded) in probability, " $\rightarrow d$ " denotes convergence in distribution, and " $\rightarrow p$ " denotes convergence in probability. 


\section{TEST STATISTIC AND ASYMPTOTIC THEORY}

Denote by $\gamma(j)=\operatorname{cov}\left(\varepsilon_{t}, \varepsilon_{t+j}\right)$. Let

$$
f(\omega)=\frac{1}{2 \pi} \sum_{j=-\infty}^{\infty} \gamma(j) e^{-i j \omega} \text { for } \omega \in[-\pi, \pi]
$$

and $F(\lambda)=\int_{0}^{\lambda} f(\omega) d \omega$ for $\lambda \in[0, \pi]$ be the spectral density function and spectral distribution function of $\varepsilon_{t}$, respectively. Note that $F(\lambda)=\sum_{j=0}^{\infty} \gamma(j) \psi_{j}(\lambda)$, where

$$
\psi_{j}(\lambda)=\left\{\begin{array}{ll}
\sin (j \lambda) / j \pi & \text { if } j \neq 0 \\
\lambda / 2 \pi & \text { if } j=0
\end{array} .\right.
$$

Then, following Shao (2011a), the sample spectral distribution function of $\varepsilon_{t}$ is

$$
F_{n}(\lambda)=\sum_{j=0}^{n-1} \widehat{\gamma}(j) \psi_{j}(\lambda)
$$

where $\widehat{\gamma}(j)=n^{-1} \sum_{t=1+|j|}^{n} \varepsilon_{t} \varepsilon_{t-|j|}$ is the sample autocovariance function of $\varepsilon_{t}$ at lag $j$. Since $F(\lambda)=\gamma(0) \psi_{0}(\lambda)$ under the null hypothesis

$H_{0}: y_{t}$ admits a weak $\operatorname{ARMA}(p, q)$ model representation as in (1),

the sample spectral distribution $F_{n}(\lambda)$ becomes $\widehat{\gamma}(0) \psi_{0}(\lambda)$ in this case. Thus, as in Shao (2011a), we consider the following Cramér von-Mises statistic

$$
\mathrm{CM}_{n}=\int_{0}^{\pi} S_{n}^{2}(\lambda) d \lambda
$$

to detect $H_{0}$, where the process

$$
S_{n}(\lambda)=\sqrt{n}\left\{F_{n}(\lambda)-\widehat{\gamma}(0) \psi_{0}(\lambda)\right\}:=\sum_{j=1}^{n-1} \sqrt{n} \widehat{\gamma}(j) \psi_{j}(\lambda)
$$

measures the distance between $F_{n}(\lambda)$ and $\widehat{\gamma}(0) \psi_{0}(\lambda)$. However, the statistic $\mathrm{CM}_{n}$ is not feasible ${ }_{85}$ because $\varepsilon_{t}$ is unobservable.

Next, let $\theta=\left(\phi_{1}, \cdots, \phi_{p}, \varphi_{1}, \cdots, \varphi_{q}\right)^{\prime} \in \Theta$ be the unknown parameter of model (1). Then, given the observations $\left\{y_{1}, \cdots, y_{n}\right\}$, we can calculate the least squares estimator (LSE) $\theta_{n}$ defined by

$$
\theta_{n}=\arg \min _{\Theta} \widetilde{L}_{n}(\theta) \text { where } \widetilde{L}_{n}(\theta)=\frac{1}{n} \sum_{t=1}^{n} \widetilde{\varepsilon}_{t}^{2}(\theta)=: \frac{1}{n} \sum_{t=1}^{n} \widetilde{l}_{t}(\theta),
$$

and $\widetilde{\varepsilon}_{t}(\theta)$ is calculated recursively by

$$
\widetilde{\varepsilon}_{t}(\theta)=y_{t}-\sum_{i=1}^{p} \phi_{i} y_{t-i}-\sum_{i=1}^{q} \varphi_{i} \widetilde{\varepsilon}_{t-i}(\theta)
$$

with $\widetilde{\varepsilon}_{0}(\theta)=\widetilde{\varepsilon}_{-1}(\theta)=\cdots=\widetilde{\varepsilon}_{-q+1}(\theta)=y_{0}=y_{-1}=\cdots=y_{-p+1}=0$. Now, by using the residual $\widetilde{\varepsilon}_{t}=\widetilde{\varepsilon}_{t}\left(\theta_{n}\right)$, we can propose a feasible Cramér von-Mises statistic as follows:

$$
\widetilde{\mathrm{CM}}_{n}=\int_{0}^{\pi} \widetilde{S}_{n}^{2}(\lambda) d \lambda
$$


where $\widetilde{S}_{n}(\lambda)=\sum_{j=1}^{n-1} \sqrt{n} \widetilde{\gamma}(j) \psi_{j}(\lambda)$ and $\widetilde{\gamma}(j)=n^{-1} \sum_{t=1+|j|}^{n} \widetilde{\varepsilon}_{t} \widetilde{\varepsilon}_{t-|j|}$.

In order to obtain the limiting distribution of $\widetilde{\mathrm{CM}}_{n}$, we regard $\widetilde{S}_{n}(\lambda)$ as a random element in the Hilbert space $L_{2}[0, \pi]$ of all square integrable functions with the inner product

$$
\langle f, g\rangle=\int_{0}^{\pi} f(\lambda) g^{c}(\lambda) d \lambda
$$

where $g^{c}(\lambda)$ denotes the complex conjugate of $g(\lambda)$. Here, $L_{2}[0, \pi]$ is endowed with the natural Borel $\sigma$-field induced by the norm $\|f\|=\langle f, f\rangle^{1 / 2}$; see Parthasarathy (1967). Since the " $\|\cdot\| "$ functional is a continuous mapping from $L_{2}[0, \pi]$ to $\mathcal{R}$, the limiting distribution of $\widetilde{\mathrm{CM}}_{n}$ follows directly from the weak convergence of $\widetilde{S}_{n}(\lambda)$ in $L_{2}[0, \pi]$; see, e.g., Politis and Romano (1994), Escanciano (2006), Shao (2011a), and many others.

Let $\varepsilon_{t}(\theta)$ be the parametric model (1), i.e., given initial values $\left\{y_{0}, y_{-1}, \cdots\right\}$ and observations $\left\{y_{1}, \cdots, y_{n}\right\}, \varepsilon_{t}(\theta)$ is iteratively constructed from

$$
\varepsilon_{t}(\theta)=y_{t}-\sum_{i=1}^{p} \phi_{i} y_{t-i}-\sum_{i=1}^{q} \varphi_{i} \varepsilon_{t-i}(\theta) .
$$

To obtain the weak convergence of $\widetilde{S}_{n}(\lambda)$ in $L_{2}[0, \pi]$, we make the following three assumptions:

Assumption 1. (i) The parametric space $\Theta \subset \mathcal{R}^{p+q}$ is compact, and the true parameter $\theta_{0}$ of model (1) belongs to the interior of $\Theta$.

(ii) For each $\theta \in \Theta, \phi(z):=1-\sum_{i=1}^{p} \phi_{i} z^{i} \neq 0$ and $\varphi(z):=1+\sum_{i=1}^{q} \varphi_{i} z^{i} \neq 0$ when $|z| \leq$ 1 , and $\phi(z)$ and $\varphi(z)$ have no common root with $\phi_{p} \neq 0$ or $\varphi_{q} \neq 0$.

Assumption 2. $\left\{y_{t}\right\}$ is strictly stationary with $E\left|y_{t}\right|^{4+2 \nu}<\infty$ and

$$
\text { (i) } \sum_{k=0}^{\infty}\left\{\alpha_{y}(k)\right\}^{\nu /(2+\nu)}<\infty
$$

for some $\nu>0$, where $\left\{\alpha_{y}(k)\right\}$ is the sequence of strong mixing coefficients of $\left\{y_{t}\right\}$;

$$
\text { (ii) } \sum_{s_{1}, s_{2}, s_{3}=-\infty}^{\infty}\left|\operatorname{cum}\left(y_{0}, y_{s_{1}}, y_{s_{2}}, y_{s_{3}}\right)\right|<\infty \text {. }
$$

Assumption 3. (i) There exists a unique interior point $\check{\theta}_{0} \in \Theta$ such that $\left\|\theta_{n}-\breve{\theta}_{0}\right\|=o_{p}(1)$.

(ii) The matrix $\Sigma=E\left[\partial^{2} l_{t}\left(\breve{\theta}_{0}\right) / \partial \theta \partial \theta^{\prime}\right]$ exists and is positive definite, where $l_{t}(\theta)=\varepsilon_{t}^{2}(\theta)$.

Assumption 1(i) is a basic set-up for model (1), and Assumption 1(ii) is the condition for the stationarity, invertibility and identifiability of model (1); see, e.g., Brockwell and Davis (1991) and Zhu and Ling (2012). Assumption 2(i) from Francq and Zakoïan (1998) is a technical condition for proving the asymptotic theory of $\theta_{n}$. In addition, the mixing condition on $y_{t}$ is valid for large classes of processes; see, e.g., Pham (1986) and Carrasco and Chen (2002). Assumption 2(ii) from Shao (2011a) is a cumulant summability condition, and a sufficient condition is given in Doukhan and León (1989), that is, there exists a $\nu_{0} \in(0,1]$ such that

$$
\sum_{k=0}^{\infty}(k+1)^{s-2}\left\{\alpha_{y}(k)\right\}^{\nu_{0} /\left(s+\nu_{0}\right)}<\infty \text { for } s=1, \cdots, 4 .
$$


Assumption 3(i) from Escanciano (2006) guarantees the weak convergence of $\theta_{n}$. Assumption 3(ii) ensures that the inverse of $\Sigma$ exists. According to Theorem 1 in Francq and Zakoïan (1998), we know that $\breve{\theta}_{0}=\theta_{0}$ under $H_{0}$. However, if $H_{0}$ fails, $\breve{\theta}_{0}$ and $\theta_{0}$ may be different.

Let $\check{\varepsilon}_{t}=\varepsilon_{t}\left(\check{\theta}_{0}\right)$ and $e_{t, j}=\check{\varepsilon}_{t} \check{\varepsilon}_{t-j}+z_{t j}$, where

$$
z_{t j}=-E\left[\frac{\partial\left(\check{\varepsilon}_{t} \check{\varepsilon}_{t-j}\right)}{\partial \theta^{\prime}}\right] \Sigma^{-1}\left[\frac{\partial l_{t}\left(\check{\theta}_{0}\right)}{\partial \theta}\right] .
$$

We are now ready to give our first main result:

TheOREm 1. Assume that Assumptions 1-3 hold. Then, as $n \rightarrow \infty$,

$$
\widetilde{S}_{n}(\lambda)-E\left\{\check{S}_{n}(\lambda)\right\} \Rightarrow S(\lambda),
$$

where “ $\Rightarrow$ " stands for weak convergence in $L_{2}[0, \pi]$ endowed with the norm metric,

$$
\breve{S}_{n}(\lambda)=\sum_{j=1}^{n-1} \sqrt{n} \check{\gamma}(j) \psi_{j}(\lambda) \text { with } \check{\gamma}(j)=n^{-1} \sum_{t=1+|j|}^{n} \check{\varepsilon}_{t} \check{\varepsilon}_{t-|j|},
$$

and $S(\lambda)$ is a Gaussian process in $C[0, \pi]$ with mean zero and covariance function

$$
\operatorname{cov}\left\{S(\lambda), S\left(\lambda^{\prime}\right)\right\}=\sum_{j=1}^{\infty} \sum_{k=1}^{\infty} \sum_{d=-\infty}^{\infty} \operatorname{cov}\left(e_{t, j}, e_{t-d, k}\right) \psi_{j}(\lambda) \psi_{k}\left(\lambda^{\prime}\right) .
$$

COROllary 1. Assume that Assumptions 1-3 hold. Then, as $n \rightarrow \infty$,

$$
\begin{aligned}
& \text { (i) } \widetilde{C M}_{n} \rightarrow{ }_{d} \int_{0}^{\pi} S^{2}(\lambda) d \lambda \text { under } H_{0} ; \\
& \text { (ii) } \frac{\widetilde{C M}_{n}}{n} \rightarrow p \sum_{j=1}^{\infty}\left[E\left(\check{\varepsilon}_{t} \breve{\varepsilon}_{t-j}\right)\right]^{2} \int_{0}^{\pi} \psi_{j}^{2}(\lambda) d \lambda .
\end{aligned}
$$

Remark 1. When $p=q=0$, the Gaussian process $S(\lambda)$ is the same as the one in Theorem 2.1 of Shao (2011a). When some $p$ or $q$ is nonzero, the Gaussian process $S(\lambda)$ depends on $z_{t j}$, which is caused by the estimation effect. This phenomenon happens not only in our case but in most specification tests.

Remark 2. When $\varepsilon_{t}$ follows a GARCH model, Ling (2007) showed that a finite fourth moment of $y_{t}$ is necessary to prove the asymptotic normality of the LSE in ARMA-GARCH models. In view of this, our moment assumption on $y_{t}$ is not restrictive.

Remark 3. In the proof of Theorem 1, we use a mixing condition of $y_{t}$ to ensure the asymptotic normality theory; see Rosenblatt (1985). Recently, an alternative way for this is to use the physical dependence condition of $y_{t}$ in Wu (2005) as done by Shao (2011a, b) and many others. In general, the physical dependence condition is implied by the geometric-moment contraction (GMC) condition defined as follows:

Definition [Wu (2005)]: Assume that $y_{t}=G\left(\cdots, \varepsilon_{t-1}, \varepsilon_{t}\right)$, where $G$ is a measurable function. Let $\left\{\varepsilon_{k}^{\prime}\right\}_{k \in \mathcal{Z}}$ be an iid copy of $\left\{\varepsilon_{k}\right\}_{k \in \mathcal{Z}}$, and $y_{t}^{\prime}=G\left(\cdots, \varepsilon_{-1}^{\prime}, \varepsilon_{0}^{\prime}, \varepsilon_{1}, \cdots, \varepsilon_{t}\right)$ be a coupled version of $y_{t}$. Then, $y_{t}$ is $\operatorname{GMC}(\alpha)$ for $\alpha>0$, if there exist $C>0$ and $\rho=\rho(\alpha) \in(0,1)$ such that $E\left(\left|y_{t}-y_{t}^{\prime}\right|^{\alpha}\right) \leq C \rho^{t}$ for $t \in \mathcal{Z}$. 
The GMC condition indicates that the process $\left\{y_{t}\right\}$ forgets its past exponentially fast, and this can not be implied from the $\alpha$-mixing condition. Shao and Wu (2007) and Shao (2011b) have verified the GMC condition for many nonlinear time series models such as GARCH models, all-pass ARMA models, bilinear models, to name a few. Particularly, if $y_{t}$ satisfies the GMC(4) condition, Assumption 2(ii) holds according to Proposition 2 in Wu and Shao (2004). Needless to say, the concepts of $\alpha$-mixing and GMC are two parallel tools to depict the dependence structure of $y_{t}$. In this paper, we mainly focus on the $\alpha$-mixing condition, and our results could be obtained similarly in the GMC context.

Remark 4. Let $r_{0}=s_{0}=2+2 \nu /(4+\nu)(\leq 4)$. Under Assumption 2(i), the Davydov's inequality in Davydov (1968) implies that

$$
\left|\operatorname{cov}\left(y_{t}, y_{t-k}\right)\right| \leq O(1)\left\|y_{t}\right\|_{r_{0}}\left\|y_{t-k}\right\|_{s_{0}}\left[\alpha_{y}(k)\right]^{1-1 / r_{0}-1 / s_{0}}
$$

for any $k \geq 0$. Thus, it follows that

$$
\sum_{k=0}^{\infty}\left|\operatorname{cov}\left(y_{t}, y_{t-k}\right)\right|^{2} \leq O(1) \sum_{k=0}^{\infty}\left[\alpha_{y}(k)\right]^{\nu /(2+\nu)}<\infty
$$

So, we know that $\sum_{k=-\infty}^{\infty}[\gamma(k)]^{2}<\infty$, and hence $\sum_{k=-\infty}^{\infty}|\gamma(k)|<\infty$, i.e., $y_{t}$ is a short memory process under Assumption 2(i).

In practice, since $\theta_{0}$ is generally unknown, one may focus on the following alternative hypothesis $H_{1}$, where

$H_{1}: y_{t}$ does not admit a weak $\operatorname{ARMA}(p, q)$ model representation as in (1) with parameter $\check{\theta}_{0}$.

Since at least one $E\left(\breve{\varepsilon}_{t} \breve{\varepsilon}_{t-j}\right) \neq 0$ under $H_{1}$, the test statistic $\widetilde{\mathrm{CM}}_{n}$ is consistent in detecting $H_{1}$ by Corollary 1(ii).

In the end, as in Shao (2011a), we consider a Pitman's local alternative as follows:

$$
H_{1 n}: f_{n}(\omega)=\frac{\gamma(0)}{2 \pi}\left(1+\frac{g(\omega)}{\sqrt{n}}\right)
$$

where $\omega \in[-\pi, \pi], g$ is a symmetric and $2 \pi$-periodic function that satisfies $\int_{-\pi}^{\pi} g(\omega) d \omega=0$.

Clearly, $f_{n}$ is a valid spectral density function, and under $H_{1 n}$,

$$
\gamma_{n}(j)=\left\{\begin{array}{ll}
\frac{\gamma(0)}{2 \pi \sqrt{n}} \int_{-\pi}^{\pi} g(\omega) e^{i j \omega} d \omega & \text { if } j \neq 0 \\
\gamma(0) & \text { if } j=0
\end{array} .\right.
$$

As in Escanciano (2006), we need one more assumption as follows:

Assumption 4. Under $H_{1 n},\left\|\theta_{n}-\theta_{0}\right\|=o_{p}(1)$ (i.e., $\theta_{0}=\breve{\theta}_{0}$ ).

Corollary 2. Assume that Assumptions 1-4 hold. Then, under $H_{1 n}$, as $n \rightarrow \infty$,

$$
\widetilde{C M}_{n} \rightarrow \int_{0}^{\pi}\{S(\lambda)+\Pi(\lambda)\}^{2} d \lambda,
$$

where $\Pi(\lambda)=\frac{\gamma(0)}{2 \pi} \int_{0}^{\lambda} g(\omega) d \omega$.

Corollary 2 shows that if the value of $\Pi(\lambda)$ deviates from zero, $\widetilde{\mathrm{CM}}_{n}$ has nontrivial power against the local alternative of order $n^{-1 / 2}$. Note that the kernel-based spectral test $T_{n}$ in 
Hong (1996) and Shao (2011b) only has nontrivial power against the local alternative of order $\left(n / m_{n}^{1 / 2}\right)^{-1 / 2}$, where

$$
T_{n}=\sum_{j=1}^{n-1} K^{2}\left(\frac{j}{m_{n}}\right) \widetilde{\rho}^{2}(j),
$$

with $\widetilde{\rho}(j)=\widetilde{\gamma}(j) / \widetilde{\gamma}(0)$ being the residual autocorrelation at lag $j, K(\cdot)$ being the kernel function satisfying Assumption 2.1 in Shao (2011b), and $m_{n}$ being the bandwidth such that $\log n=$ $o\left(m_{n}\right)$ and $m_{n}=o\left(n^{1 / 2}\right)$. However, this does not guarantee that $\widetilde{\mathrm{CM}}_{n}$ is always more powerful than $T_{n}$ under $H_{1 n}$. To see the reason, on one hand, by (A20) in the Appendix, we have

$$
\Pi(\lambda) \approx \sum_{j=1}^{n} \sqrt{n} \gamma_{n}(j) \psi_{j}(\lambda)=\sum_{j=1}^{n} \sqrt{n} \gamma_{n}(j) \frac{\sin (j \pi)}{j \pi},
$$

from which we know that the impact of $\gamma_{n}(j)$ to $\Pi(\lambda)$ is proportional to $j^{-1}$. So, it implies that if $\gamma_{n}(j)$ is only significantly different from zero at large lag $j$, the value of $\Pi(\lambda)$ may not deviate significantly from zero, and hence this will cause a low local power of $\widetilde{\mathrm{CM}}_{n}$. On the other hand, the local power of $T_{n}$ tends to be proportional to $\left\|g / m_{n}^{1 / 4}\right\|$ under $H_{1 n}$ (see Theorem 4 in Hong (1996)). From (4), we know that the value of $\|g\|$ becomes large when the value of $\gamma_{n}(j)$ is significantly different from zero at any lag $j$. Thus, if the value of $m_{n}$ is not big enough, $T_{n}$ will not be deficient in local power even when $\gamma_{n}(j)$ is only significantly different from zero at large lag $j$. Generally speaking, under $H_{1 n}, \widetilde{\mathrm{CM}}_{n}$ may be locally more (or less) powerful than $T_{n}$, when $\gamma_{n}(j)$ is significantly different from zero at small (or large) lag $j$; see, e.g., the simulation results for Examples 1 and 2 in Section 4 below. Similar phenomenon has been well documented by Eubank and LaRiccia (1992) and Paparoditis (2001). Moreover, if we consider Rosenblatt's (1975) sharp peak local alternative, the asymptotic theory of $\widetilde{\mathrm{CM}}_{n}$ and $T_{n}$ is still unclear. The pioneering work in Ghosh and Huang (1991) and Paparoditis (2001) may be extended to both tests, and we leave it for future study.

\section{BOOTSTRAPPED CRITICAL VALUES}

Since the limiting distribution of $\widetilde{\mathrm{CM}}_{n}$ depends on the unknown model parameters and unknown structure of dependent innovation, we use a block-wise random weighting (BRW) method to bootstrap its critical values. The detailed steps are as follows:

1. Set a block size $b_{n}$, such that $1 \leq b_{n}<n$. Denote the blocks by $B_{s}=\left\{(s-1) b_{n}+\right.$ $\left.1, \cdots, s b_{n}\right\}$ for $s=1, \cdots, L_{n}$, where $L_{n}=n / b_{n}$ is assumed to be an integer for the convenience of presentation.

2. Generate a sequence of positive i.i.d. random variables $\left\{\delta_{1}, \cdots, \delta_{L_{n}}\right\}$, independent of the data, from a common distribution $W$, where $E(W)=1$ and $\operatorname{var}(W)=1$. Define the random weights $w_{t}^{*}=\delta_{s}$, if $t \in B_{s}$, for $t=1, \cdots, n$. Calculate $\theta_{n}^{*}$ via

$$
\theta_{n}^{*}=\arg \min _{\Theta} \widetilde{L}_{n}^{*}(\theta), \text { where } \widetilde{L}_{n}^{*}(\theta)=\frac{1}{n} \sum_{t=1}^{n} w_{t}^{*} \widetilde{\varepsilon}_{t}^{2}(\theta)=: \frac{1}{n} \sum_{t=1}^{n} \tilde{l}_{t}^{*}(\theta) .
$$

3. Let $\widetilde{\varepsilon}_{t}^{*}=\widetilde{\varepsilon}_{t}\left(\theta_{n}^{*}\right)$ for $t=1, \cdots, n$, with $\widetilde{\varepsilon}_{t}(\theta)$ being defined as in (2), and

$$
\widetilde{S}_{n}^{*}(\lambda)=\sum_{j=1}^{n-1} \sqrt{n} \widetilde{\gamma}^{*}(j) \psi_{j}(\lambda) \text { with } \widetilde{\gamma}^{*}(j)=\frac{1}{n} \sum_{t=1+j}^{n} w_{t}^{*} \widetilde{\varepsilon}_{t}^{*} \widetilde{\varepsilon}_{t-j}^{*} .
$$


Define the bootstrapped process $\Delta_{n}(\lambda)=\widetilde{S}_{n}^{*}(\lambda)-\widetilde{S}_{n}(\lambda)-\widetilde{Z}_{n}(\lambda)$, where

$$
\widetilde{Z}_{n}(\lambda)=\sum_{j=1}^{n-1}\left\{\frac{1}{\sqrt{n}} \sum_{t=1+j}^{n}\left[\left(w_{t}^{*}-1\right) \widetilde{\gamma}(j)\right]\right\} \psi_{j}(\lambda) .
$$

4. Compute the bootstrapped test statistic $\widetilde{\mathrm{CM}}_{n}^{*}=\int_{0}^{\pi}\left\{\Delta_{n}(\lambda)\right\}^{2} d \lambda$.

THEOREM 2. Assume that (a) Assumptions 1-3 hold; (b) $E\left|y_{t}\right|^{8+4 \nu}<\infty$ for some $\nu>0$ and $\lim _{k \rightarrow \infty} k^{2}\left[\alpha_{y}(k)\right]^{\nu /(2+\nu)}=0 ;(c) b_{n}^{-1}=o(1)$ and $b_{n}=o\left(n^{1 / 3}\right) ;(d) E\left(w_{t}^{*}\right)^{4}<\infty$. Then, as $n \rightarrow \infty$,

$$
\text { (i) } d_{\omega}\left[\mathcal{L}\left\{\Delta_{n}(\lambda) \mid \chi_{n}\right\}, \mathcal{L}\{S(\lambda)\}\right] \rightarrow_{p} 0
$$

(ii) consequently,

$$
\widetilde{C M}_{n}^{*} \rightarrow_{d} \int_{0}^{\pi} S^{2}(\lambda) d \lambda \text { in probability. }
$$

Remark 5. An exponentially fast decaying $\alpha_{y}(k)$ is sufficient for the condition on $\alpha_{y}(k)$ in Theorem 2 to hold.

Compared to the conditions in Shao (2011a), our conditions in Theorem 2 are stronger. This is a price we pay for not assuming a stronger cumulant summability condition:

$$
\sum_{s_{1}, \cdots, s_{K}=-\infty}^{\infty}\left|s_{k}\right|\left|\operatorname{cum}\left(y_{0}, y_{s_{1}} \cdots, y_{s_{K}}\right)\right|<\infty, k=1, \cdots, K,
$$


for $K=1, \cdots, 7$. Although (7) is implied by the $\mathrm{GMC}(8)$ condition of $y_{t}$ according to Proposition 2 of Wu and Shao (2004), a sufficient condition for (7) in the context of $\alpha$-mixing condition is still unknown. If (7) holds, following a similar proof in Shao (2011a, p.221-222), we can easily show that Theorem 2 holds under some weaker conditions. We summarize it in the following theorem:

THEOREM 3. Assume that (a) Assumptions 1-3 and (7) hold; (b) E $y_{t}^{8}<\infty$; (c) $b_{n}^{-1}=o(1)$ and $(\log n) b_{n}=o(n) ;(d) E\left(w_{t}^{*}\right)^{4}<\infty$. Then, the conclusions in Theorem 2 hold.

Remark 6. Theorems 2-3 guarantee that when $J$ is large, our bootstrapped critical values from the BRW method are valid for $\widetilde{\mathrm{CM}}_{n}$ under the null or the alternative hypothesis. The reason why our bootstrap method works is probably because the conditional distribution of $\sqrt{n}\left(\theta_{n}^{*}-\theta_{n}\right)$ can always well mimic the distribution of $\sqrt{n}\left(\theta_{n}-\breve{\theta}_{0}\right)$, and this guarantees that we can handle the estimation effect successfully without generating any pseudo series. To see it clearly, from Lemma A3(ii) in the Appendix, we have

$$
\sqrt{n}\left(\theta_{n}-\check{\theta}_{0}\right) \rightarrow_{d} N(0, V) \text { as } n \rightarrow \infty,
$$

where $V=\Sigma^{-1} \Omega \Sigma^{-1}$ and

$$
\Omega=\lim _{n \rightarrow \infty} \operatorname{var}\left[\frac{1}{\sqrt{n}} \sum_{t=1}^{n} \frac{\partial l_{t}\left(\check{\theta}_{0}\right)}{\partial \theta}\right]
$$

is well defined by Lemma 3 in Francq and Zakoïan (1998). By (A39) in the Appendix, it is not hard to see that under the conditions of Theorem 2 or 3, we have

$$
\mathcal{L}\left\{\sqrt{n}\left(\theta_{n}^{*}-\theta_{n}\right) \mid \chi_{n}\right\} \rightarrow_{d} N(0, V) \text { in probability as } n \rightarrow \infty .
$$

By (8) and (10), it follows that we can estimate $V$ by $\widetilde{V}_{n}$ even when model (1) is misspecified (i.e., $\widetilde{\theta}_{0} \neq \theta_{0}$ ) under the alternative hypothesis, where

$$
\widetilde{V}_{n}:=\text { sample variance-covariance matrix of }\left\{\sqrt{n}\left(\theta_{1 n}^{*}-\theta_{n}\right), \cdots, \sqrt{n}\left(\theta_{J n}^{*}-\theta_{n}\right)\right\},
$$

and $\theta_{i n}^{*}$ for $i=1, \cdots, J$ is calculated from step 2 in our BRW procedure. Particularly, by using $\widetilde{V}_{n}$, the classical Wald test is now applicable for model (1).

Remark 7. Since $\varepsilon_{t}$ is unobservable with unknown dependent structure beyond MDS, another intuitive way to bootstrap the null distribution of $\widetilde{\mathrm{CM}}_{n}$ is using the residual-based block bootstrap in Paparoditis and Politis (2003) or the residual-based stationary bootstrap in Parker, Paparoditis, and Politis (2006). Both methods have been used for unit root testing. Their ideas are to generate pseudo series $\left\{y_{t}^{* *}\right\}$ from model (1) with parameter $\theta_{n}$, where the error sequence is obtained by sampling blocks of the residual series $\left\{\widetilde{\varepsilon}_{t}\right\}$ randomly with replacement. Then, we can calculate the bootstrapped samples $\left\{\widetilde{\mathrm{CM}}_{i n}^{* *}\right\}_{i=1}^{J}$ from the bootstrapped residuals $\left\{\widetilde{\varepsilon}_{t}^{* *}\left(\theta_{n}^{* *}\right)\right\}$, where $\theta_{n}^{* *}=$ $\arg \min _{\Theta} n^{-1} \sum_{t=1}^{n}\left[\widetilde{\varepsilon}_{t}^{* *}(\theta)\right]^{2}$, and

$$
\widetilde{\varepsilon}_{t}^{* *}(\theta)=y_{t}^{* *}-\sum_{i=1}^{p} \phi_{i} y_{t-i}^{* *}-\sum_{i=1}^{q} \psi_{i} \widetilde{\varepsilon}_{t}^{* *}(\theta) .
$$

However, $y_{t}^{* *}$ may not well mimic $y_{t}$, because the residual series $\left\{\widetilde{\varepsilon}_{t}\right\}$ is correlated under the alternative hypothesis; see also Jentsch, Politis, and Paparoditis (2014) on a similar phenomenon for integrated processes. Based on this concern, the empirical distribution of $\left\{\widehat{\mathrm{CM}}_{i n}^{* *}\right\}$ can not 
properly mimic the null distribution, and hence we do not follow the residual-based block methods in this paper.

Remark 8. In order to implement $\widetilde{\mathrm{CM}}_{n}$ in practice, we need to first select the orders $p$ and $q$. When $\left\{\varepsilon_{t}\right\}$ is an iid sequence, this can be done by many well-known information criteria such as AIC, BIC, or EACF; see, e.g., Tsay and Tiao (1984). When $\left\{\varepsilon_{t}\right\}$ is an MDS, Chen, Min, and Chen (2013) proposed some order determination schemes based on the ACF and PACF of $y_{t}$. When $\left\{\varepsilon_{t}\right\}$ is an uncorrelated sequence, there is no valid method to select the orders so far, and we suggest using a Wald test $\Xi_{n}$ to choose the orders by detecting the hypothesis $\Gamma \check{\theta}_{0}=0_{s \times 1}$, where $\Gamma$ is a $s \times(p+q)$ constant matrix with rank $s$, and

$$
\Xi_{n}=n\left(\Gamma \theta_{n}\right)^{\prime}\left(\Gamma \widetilde{V}_{n} \Gamma^{\prime}\right)^{-1}\left(\Gamma \theta_{n}\right)
$$

with $\widetilde{V}_{n}$ being defined as in (11). By using $\Xi_{n}$, it is now possible to reduce the orders from a vast fitted model, and this could enhance the power of our test.

Remark 9. By a repetitive but even simpler proof as in the Appendix, we can show that Theorems 2-3 hold if $b_{n}=1$ when $\varepsilon_{t}$ is an MDS. However, when $\varepsilon_{t}$ is not an MDS, we need a block technique (i.e., $b_{n} \neq 1$ ) to capture the dependence of $\varepsilon_{t}$ beyond MDS; see, e.g., Romano and Thombs (1996), Horowitz, Lobato, Nankervis, and Savin (2006), and Shao (2011a). Compared to the proof of Theorem 1 in the unconditional case, the proofs of Theorems 2-3 in the conditional case follow the same idea but with more nontrivial proofs caused by the block technique.

Finally, it is worth noting that Theorem 2 requires a stronger condition for $b_{n}$ than Theorem 3. This demonstrates that if we allow for a more general structure of $y_{t}$, we may suffer from a smaller valid range of $b_{n}$. Hence, there is a tradeoff between the dependence structure of $y_{t}$ and the theoretical valid range of $b_{n}$. Also, we should highlight that the often used block-wise wild bootstrap method applies the random weight $w_{t}^{*}$ to the residual $\widetilde{\varepsilon}_{t}$ directly, but this method may fail in some cases as shown in Section 4.3 of Brüggemann, Jentsch, and Trenkler (2014). Our BRW method essentially applies the random weight $w_{t}^{*}$ to the product of the residual $\widetilde{\varepsilon}_{t} \widetilde{\varepsilon}_{t+j}$ $(j \geq 1)$, and so it is different from the often used one. As one referee pointed out, this feature of our BRW method may lead to a robust size performance in terms of $b_{n}$, especially when the dependence structure of $\varepsilon_{t} \varepsilon_{t+j}(j \geq 1)$ is weak; see, e.g., Figure 1 in Section 4 below. Nevertheless, how to select the optimal $b_{n}$ under certain "criterion" is unknown up to now. This is a familiar problem with all blocking methods. The heuristic work in Hall, Horowitz, and Jing (1995) and Politis, Romano, and Wolf (1999) may be extended in this case, and we leave it for future study.

\section{Simulation STUdies}

In this section, we examine the finite-sample performance of $\widetilde{\mathrm{CM}}_{n}$ for several weak ARMA models. As a comparison, we also consider the kernel-based test $T_{n}$ in (5). Under $H_{0}$ and certain conditions, Shao (2011b) showed that

$$
\frac{n T_{n}-m_{n} C(K)}{\sqrt{2 m_{n} D(K)}} \rightarrow_{d} N(0,1) \text { as } n \rightarrow \infty,
$$

where $C(K)=\int_{0}^{\infty} K^{2}(x) d x$ and $D(K)=\int_{0}^{\infty} K^{4}(x) d x$ with the kernel function $K(\cdot)$ and the bandwidth $m_{n}$ being chosen as in (5). So, we reject $H_{0}$ at the significance level $\alpha$, if $n T_{n}>\sqrt{2 m_{n} D(K)} c_{\alpha}+m_{n} C(K)$, where $c_{\alpha}$ is the $(1-\alpha)$-th percentile of $N(0,1)$. Under $H_{1}$ and certain conditions, Shao (2011b) proved that $P\left(n T_{n}>\sqrt{2 m_{n} D(K)} c_{\alpha}+m_{n} C(K)\right)=1$ as $n \rightarrow \infty$, and hence this spectral test is consistent (see also Hong (1996)). 
Next, we introduce our basic set-up. In all calculations, we generate 1000 replications of sample size $n=400$ and 1000 from each specified model in Examples 1-4 below, and choose the significance level $\alpha=1 \%, 5 \%$ or $10 \%$. For $\widetilde{\mathrm{CM}}_{n}$, we use 500 bootstrap samples in each replication with block size $b_{n}=n^{1 / 5}, 2 n^{1 / 5}, \sqrt{n} / 2, \sqrt{n}$ or $2 \sqrt{n}$ to obtain its corresponding critical value for every aforementioned significance level $\alpha$. These choices of set-up deliver $b_{n}=3,6,10,20,40$ for $n=400$ and 3, 7, 15,31, 63 for $n=1000$. Here, $\delta_{t}$ is employed from the following Bernoulli distribution:

$$
P\left(\delta_{t}=\frac{3-\sqrt{5}}{2}\right)=\frac{1+\sqrt{5}}{2 \sqrt{5}} \text { and } P\left(\delta_{t}=\frac{3+\sqrt{5}}{2}\right)=1-\frac{1+\sqrt{5}}{2 \sqrt{5}},
$$

although other choices like the standard exponential distribution are also suitable for $\delta_{t}$. For $T_{n}$, we use the Parzen kernel $K(x)$ defined as

$$
K(x)= \begin{cases}1-6 x^{2}+6|x|^{3} & \text { for } 0 \leq|x| \leq 1 / 2 \\ 2(1-|x|)^{3} & \text { for } 1 / 2 \leq|x| \leq 1, \\ 0 & \text { otherwise. }\end{cases}
$$

In general, since there is no clear objective procedure for optimally choosing the bandwidth $m_{n}$, we carry out the calculation for $m_{n}=1, \cdots, 20$ when $n=400$ and $1, \cdots, 32$ when $n=1000$. In most cases of $m_{n}$, we find that the sizes of $T_{n}$ are distorted (see Figure 1 below). Hence, only the results in which the sizes are close to their nominal ones are reported, although the corresponding choices of $m_{n}$ may not be optimal in some sense.

Example 1. Consider the following weak $\operatorname{ARMA}(1,1)$ model:

$$
y_{t}=\kappa y_{t-1}+0.8 \varepsilon_{t-1}+\varepsilon_{t} \text { and } \varepsilon_{t}=\eta_{t}^{2} \eta_{t-1},
$$

where $\eta_{t}$ is a sequence of iid $\mathrm{N}(0,1)$ random variables, and $\kappa \in\{0.0,0.1,0.2,0.3,0.4\}$. Clearly, $\varepsilon_{t}$ in (12) are uncorrelated but non-MDS. Next, we use $\widetilde{\mathrm{CM}}_{n}$ and $T_{n}$ to detect whether a weak MA(1) model is adequate to fit the data sample generated from model (12). The empirical power and sizes of both tests are reported in Table 1, and the sizes correspond to the cases where $\kappa=0.0$.

Example 2. Consider the following weak AR(2) model:

$$
y_{t}=0.5 y_{t-1}+\kappa y_{t-2}+\varepsilon_{t} \text { and } \varepsilon_{t}=\eta_{t}^{2} \eta_{t-1},
$$

where $\eta_{t}$ is a sequence of iid $\mathrm{N}(0,1)$ random variables, and $\kappa \in\{0.0,0.1,0.2,0.3,0.4\}$. We use $\widetilde{\mathrm{CM}}_{n}$ and $T_{n}$ to detect whether a weak AR(1) model is adequate to fit the data sample generated from model (13). The empirical power and sizes of both tests are reported in Table 2, and the sizes correspond to the cases where $\kappa=0.0$.

Example 3. Consider the following switching-regime Markov model (see, e.g., Hamilton (1994)):

$$
y_{t}=\kappa y_{t-1}+\eta_{t}+\left(0.2+0.3 \Delta_{t}\right) \eta_{t-1},
$$

where $\Delta_{t}$ is a sequence of Bernoulli random variables with $P\left(\Delta_{t}=0\right)=1 / 3$ and $P\left(\Delta_{t}=1\right)=$ $2 / 3, \eta_{t}$ is a sequence of iid $\mathrm{N}(0,1)$ random variables, and $\kappa \in\{0.0,0.05,0.1,0.15,0.2\}$. Here, we assume that $\Delta_{t}$ and $\eta_{t}$ are independent. When $\kappa=0.0$, Francq and Zakoïan (1998) showed that model (14) admits a weak MA(1) representation: $y_{t}=\varepsilon_{t}+\varphi \varepsilon_{t-1}$, where $\varepsilon_{t}$ are uncorrelated but non-MDS. Thus, we can use $\widetilde{\mathrm{CM}}_{n}$ and $T_{n}$ to detect whether a weak MA(1) model is adequate 
to fit the data sample generated from model (14). The empirical power and sizes of both tests are reported in Table 3, and the sizes correspond to the cases where $\kappa=0.0$.

Table 1. Empirical sizes and power $(\times 100)$ for $\widetilde{C M}_{n}$ and $T_{n}$ in model (12).

\begin{tabular}{|c|c|c|c|c|c|c|c|c|c|c|c|c|c|c|c|c|c|}
\hline \multirow[b]{2}{*}{ Tests } & \multirow[b]{2}{*}{$n$} & \multirow[b]{2}{*}{$b_{n}\left(m_{n}\right)$} & \multicolumn{3}{|c|}{$\kappa=0.0$} & \multicolumn{3}{|c|}{$\kappa=0.1$} & \multicolumn{3}{|c|}{$\kappa=0.2$} & \multicolumn{3}{|c|}{$\kappa=0.3$} & \multicolumn{3}{|c|}{$\kappa=0.4$} \\
\hline & & & $1 \%$ & $5 \%$ & $10 \%$ & $1 \%$ & $5 \%$ & $10 \%$ & $1 \%$ & $5 \%$ & $10 \%$ & $1 \%$ & $5 \%$ & $10 \%$ & $1 \%$ & $5 \%$ & $10 \%$ \\
\hline \multirow[t]{5}{*}{$\widehat{\mathrm{CM}}_{n}$} & 400 & 3 & 1.3 & 6.8 & 12.5 & 3.9 & 14.1 & 26.0 & 22.0 & 49.0 & 64.4 & 54.9 & 80.2 & 89.1 & 80.1 & 93.7 & 96.8 \\
\hline & & 6 & 1.1 & 5.5 & 11.5 & 3.3 & 14.0 & 26.5 & 19.9 & 44.1 & 59.7 & 50.2 & 77.8 & 87.3 & 73.2 & 91.2 & 95.5 \\
\hline & & 10 & 1.6 & 5.5 & 10.9 & 4.2 & 15.3 & 27.1 & 22.0 & 47.3 & 60.7 & 49.6 & 75.6 & 87.1 & 68.6 & 88.0 & 95.6 \\
\hline & & 20 & 1.3 & 6.6 & 13.3 & 5.4 & 17.1 & 26.2 & 21.8 & 46.8 & 59.7 & 47.9 & 72.4 & 82.7 & 64.9 & 85.7 & 93.7 \\
\hline & & 40 & 3.2 & 7.8 & 13.3 & 8.4 & 16.8 & 25.0 & 25.1 & 44.3 & 56.4 & 48.5 & 68.4 & 80.1 & 63.8 & 80.5 & 89.9 \\
\hline \multirow[t]{2}{*}{$T_{n}$} & & 3 & 1.4 & 2.0 & 3.8 & 8.9 & 12.9 & 16.6 & 37.4 & 46.5 & 52.1 & 80.2 & 86.0 & 89.3 & 97.1 & 98.3 & 98.6 \\
\hline & & 4 & 3.1 & 6.6 & 8.2 & 15.5 & 20.7 & 24.6 & 53.8 & 61.4 & 65.8 & 88.4 & 91.2 & 92.9 & 98.1 & 99.0 & 99.5 \\
\hline \multirow[t]{5}{*}{$\overline{\mathrm{CM}}_{n}$} & 1000 & 3 & 1.2 & 5.1 & 11.6 & 13.2 & 35.6 & 48.1 & 63.8 & 82.7 & 88.8 & 94.4 & 98.4 & 99.2 & 99.1 & 99.8 & 99.9 \\
\hline & & 7 & 1.0 & 4.3 & 9.3 & 13.9 & 31.9 & 46.0 & 60.1 & 82.1 & 89.6 & 93.5 & 97.8 & 99.2 & 98.9 & 99.8 & 99.9 \\
\hline & & 15 & 1.2 & 5.3 & 11.8 & 13.8 & 33.4 & 44.8 & 62.6 & 82.7 & 90.5 & 91.5 & 97.8 & 99.0 & 97.9 & 99.7 & 99.8 \\
\hline & & 31 & 0.9 & 6.2 & 12.5 & 13.2 & 34.3 & 47.9 & 62.9 & 83.9 & 91.1 & 90.2 & 98.7 & 99.7 & 94.6 & 99.2 & 99.8 \\
\hline & & 63 & 2.1 & 6.3 & 11.7 & 17.1 & 31.6 & 46.2 & 65.7 & 82.3 & 88.4 & 86.5 & 95.8 & 97.9 & 88.5 & 96.6 & 99.0 \\
\hline \multirow[t]{2}{*}{$T_{n}$} & & 3 & 2.9 & 4.9 & 6.2 & 21.5 & 30.2 & 35.5 & 79.3 & 84.1 & 86.7 & 98.9 & 99.5 & 99.7 & 100 & 100 & 100 \\
\hline & & 4 & 5.4 & 8.2 & 11.1 & 33.0 & 41.2 & 46.2 & 87.3 & 91.2 & 92.6 & 99.9 & 100 & 100 & 100 & 100 & 100 \\
\hline
\end{tabular}

Table 2. Empirical sizes and power $(\times 100)$ for $\widetilde{C M}_{n}$ and $T_{n}$ in model (13).

\begin{tabular}{|c|c|c|c|c|c|c|c|c|c|c|c|c|c|c|c|c|c|}
\hline \multirow[b]{2}{*}{ Tests } & \multirow[b]{2}{*}{$n$} & \multirow[b]{2}{*}{$b_{n}\left(m_{n}\right)$} & \multicolumn{3}{|c|}{$\kappa=0.0$} & \multicolumn{3}{|c|}{$\kappa=0.1$} & \multicolumn{3}{|c|}{$\kappa=0.2$} & \multicolumn{3}{|c|}{$\kappa=0.3$} & \multicolumn{3}{|c|}{$\kappa=0.4$} \\
\hline & & & $1 \%$ & $5 \%$ & $10 \%$ & $1 \%$ & $5 \%$ & $10 \%$ & $1 \%$ & $5 \%$ & $10 \%$ & $1 \%$ & $5 \%$ & $10 \%$ & $1 \%$ & $5 \%$ & $10 \%$ \\
\hline \multirow[t]{5}{*}{$\widehat{\mathrm{CM}}_{n}$} & 400 & 3 & 0.5 & 4.6 & 9.9 & 19.5 & 35.9 & 46.8 & 63.7 & 80.9 & 86.2 & 89.6 & 95.2 & 96.9 & 97.0 & 98.7 & 99.2 \\
\hline & & 6 & 2.0 & 4.4 & 10.1 & 18.0 & 35.2 & 46.0 & 61.4 & 79.0 & 86.0 & 86.2 & 93.3 & 95.9 & 94.4 & 98.5 & 99.3 \\
\hline & & 10 & 1.1 & 4.8 & 10.4 & 20.0 & 39.8 & 51.5 & 66.3 & 83.4 & 88.6 & 86.7 & 95.1 & 97.1 & 95.6 & 98.5 & 99.0 \\
\hline & & 20 & 1.6 & 6.1 & 12.7 & 22.6 & 41.4 & 52.1 & 65.6 & 81.5 & 87.8 & 87.4 & 96.1 & 97.5 & 94.1 & 98.5 & 99.1 \\
\hline & & 40 & 2.6 & 6.4 & 13.1 & 25.4 & 37.7 & 47.3 & 64.2 & 78.7 & 84.9 & 85.4 & 94.8 & 97.4 & 92.4 & 98.3 & 98.8 \\
\hline \multirow[t]{2}{*}{$T_{n}$} & & 10 & 1.9 & 3.4 & 4.9 & 15.1 & 23.6 & 28.5 & 72.2 & 78.2 & 82.5 & 97.0 & 97.7 & 98.0 & 99.9 & 99.9 & 99.9 \\
\hline & & 15 & 2.4 & 4.3 & 6.8 & 16.9 & 24.6 & 30.4 & 72.5 & 79.5 & 82.7 & 96.8 & 98.4 & 98.7 & 99.9 & 99.9 & 100 \\
\hline \multirow[t]{5}{*}{$\widetilde{\mathrm{CM}}_{n}$} & 1000 & 3 & 1.3 & 5.6 & 11.1 & 45.2 & 65.7 & 75.3 & 93.8 & 98.2 & 98.6 & 99.6 & 99.9 & 100 & 100 & 100 & 100 \\
\hline & & 7 & 1.1 & 6.3 & 10.8 & 48.7 & 67.9 & 74.7 & 93.2 & 97.1 & 98.4 & 99.7 & 99.9 & 99.9 & 99.9 & 100 & 100 \\
\hline & & 15 & 1.2 & 6.4 & 12.2 & 48.3 & 67.1 & 75.9 & 92.4 & 97.6 & 98.7 & 99.7 & 99.9 & 100 & 99.7 & 100 & 100 \\
\hline & & 31 & 1.2 & 5.2 & 11.7 & 48.1 & 66.6 & 74.9 & 92.6 & 96.6 & 98.0 & 99.3 & 99.9 & 99.9 & 99.8 & 100 & 100 \\
\hline & & 63 & 1.6 & 6.4 & 11.5 & 49.9 & 66.7 & 75.5 & 94.6 & 97.8 & 98.8 & 99.4 & 99.8 & 100 & 99.8 & 100 & 100 \\
\hline \multirow[t]{2}{*}{$T_{n}$} & & 9 & 2.0 & 3.7 & 5.6 & 44.7 & 56.0 & 62.5 & 97.8 & 98.9 & 99.4 & 100 & 100 & 100 & 100 & 100 & 100 \\
\hline & & 13 & 2.9 & 5.4 & 7.5 & 51.0 & 60.3 & 64.9 & 98.1 & 99.0 & 99.3 & 100 & 100 & 100 & 100 & 100 & 100 \\
\hline
\end{tabular}

Example 4. Consider the following bilinear model (see, e.g., Granger and Andersen (1978) 305 and Pham (1986)):

$$
y_{t}=\kappa \eta_{t-1}+\eta_{t}+0.2 y_{t-1} \eta_{t-2}
$$


where $\eta_{t}$ is a sequence of iid $\mathrm{N}(0,1)$ random variables, and $\kappa \in\{0.0,0.05,0.1,0.15,0.2\}$. When $\kappa=0.0$, Francq and Zakoïan (1998) showed that model (15) admits a weak MA(3) representation: $y_{t}=\varepsilon_{t}+\varphi \varepsilon_{t-3}$, where $\varepsilon_{t}$ are uncorrelated but non-MDS. Thus, we can use $\widetilde{\mathrm{CM}}_{n}$ and $T_{n}$ to detect whether a weak MA(3) model is adequate to fit the data sample generated from model (15). The empirical power and sizes of both tests are reported in Table 4, and the sizes correspond to the cases where $\kappa=0.0$.

Table 3. Empirical sizes and power $(\times 100)$ for $\widetilde{C M}_{n}$ and $T_{n}$ in model (14).

\begin{tabular}{|c|c|c|c|c|c|c|c|c|c|c|c|c|c|c|c|c|c|}
\hline \multirow[b]{2}{*}{ Tests } & \multirow[b]{2}{*}{$n$} & \multirow[b]{2}{*}{$b_{n}\left(m_{n}\right)$} & \multicolumn{3}{|c|}{$\kappa=0.0$} & \multicolumn{3}{|c|}{$\kappa=0.05$} & \multicolumn{3}{|c|}{$\kappa=0.1$} & \multicolumn{3}{|c|}{$\kappa=0.15$} & \multicolumn{3}{|c|}{$\kappa=0.2$} \\
\hline & & & $1 \%$ & $5 \%$ & $10 \%$ & $1 \%$ & $5 \%$ & $10 \%$ & $1 \%$ & $5 \%$ & $10 \%$ & $1 \%$ & $5 \%$ & $10 \%$ & $1 \%$ & $5 \%$ & $10 \%$ \\
\hline \multirow[t]{5}{*}{$\mathrm{CM}_{n}$} & 400 & 3 & 1.1 & 5.4 & 10.4 & 1.9 & 8.1 & 13.9 & 4.2 & 14.2 & 22.4 & 12.7 & 32.7 & 44.1 & 29.5 & 53.8 & 65.6 \\
\hline & & 6 & 1.7 & 5.7 & 12.4 & 2.0 & 7.3 & 14.4 & 3.7 & 13.5 & 22.2 & 14.8 & 32.5 & 45.5 & 31.6 & 55.2 & 67.9 \\
\hline & & 10 & 1.7 & 6.9 & 11.8 & 2.0 & 7.6 & 13.6 & 4.8 & 13.7 & 21.5 & 15.0 & 32.0 & 43.4 & 31.8 & 55.4 & 66.8 \\
\hline & & 20 & 2.4 & 7.1 & 12.1 & 3.1 & 9.0 & 15.2 & 6.7 & 14.8 & 23.9 & 16.9 & 32.3 & 43.4 & 33.9 & 53.3 & 65.3 \\
\hline & & 40 & 3.6 & 7.8 & 13.0 & 4.6 & 10.6 & 18.6 & 9.8 & 19.1 & 28.9 & 21.9 & 36.9 & 47.7 & 40.0 & 57.6 & 69.5 \\
\hline \multirow[t]{2}{*}{$T_{n}$} & & 19 & 0.7 & 1.9 & 3.3 & 0.4 & 2.4 & 3.7 & 1.4 & 3.6 & 6.1 & 6.3 & 11.3 & 16.3 & 19.8 & 28.7 & 35.5 \\
\hline & & 20 & 0.9 & 2.1 & 3.4 & 0.8 & 2.3 & 4.4 & 2.2 & 4.8 & 8.3 & 7.2 & 13.7 & 17.6 & 16.7 & 28.0 & 34.7 \\
\hline \multirow[t]{5}{*}{$\widehat{\mathrm{CM}}_{n}$} & 1000 & 3 & 0.9 & 5.8 & 10.8 & 2.7 & 9.5 & 17.3 & 15.2 & 33.4 & 44.9 & 39.6 & 63.1 & 75.2 & 79.7 & 91.6 & 94.9 \\
\hline & & 7 & 1.6 & 5.1 & 10.5 & 4.6 & 10.9 & 17.5 & 14.5 & 29.8 & 42.1 & 40.9 & 63.6 & 75.1 & 79.2 & 91.3 & 95.7 \\
\hline & & 15 & 1.3 & 4.7 & 10.1 & 3.9 & 11.2 & 18.4 & 14.7 & 32.5 & 44.3 & 43.8 & 65.7 & 74.8 & 79.2 & 90.8 & 95.1 \\
\hline & & 31 & 1.7 & 6.1 & 10.6 & 4.2 & 11.4 & 17.3 & 16.5 & 33.9 & 45.1 & 47.4 & 69.4 & 79.5 & 79.1 & 90.5 & 94.7 \\
\hline & & 63 & 3.7 & 8.9 & 13.6 & 4.0 & 11.5 & 18.6 & 20.3 & 36.1 & 46.7 & 48.5 & 67.1 & 75.4 & 81.4 & 91.9 & 95.5 \\
\hline \multirow[t]{2}{*}{$T_{n}$} & & 21 & 0.9 & 2.4 & 4.0 & 1.9 & 4.0 & 6.5 & 7.7 & 12.7 & 17.2 & 24.4 & 37.0 & 44.5 & 61.7 & 74.8 & 79.6 \\
\hline & & 22 & 1.1 & 2.5 & 4.9 & 1.6 & 3.9 & 5.7 & 6.0 & 11.3 & 15.4 & 24.2 & 35.9 & 44.7 & 60.6 & 73.8 & 80.6 \\
\hline
\end{tabular}

Table 4. Empirical sizes and power $(\times 100)$ for $\widetilde{C M}_{n}$ and $T_{n}$ in model (15).

\begin{tabular}{|c|c|c|c|c|c|c|c|c|c|c|c|c|c|c|c|c|c|}
\hline \multirow[b]{2}{*}{ Tests } & \multirow[b]{2}{*}{$n$} & \multirow[b]{2}{*}{$b_{n}\left(m_{n}\right)$} & \multicolumn{3}{|c|}{$\kappa=0.0$} & \multicolumn{3}{|c|}{$\kappa=0.05$} & \multicolumn{3}{|c|}{$\kappa=0.1$} & \multicolumn{3}{|c|}{$\kappa=0.15$} & \multicolumn{3}{|c|}{$\kappa=0.2$} \\
\hline & & & $1 \%$ & $5 \%$ & $10 \%$ & $1 \%$ & $5 \%$ & $10 \%$ & $1 \%$ & $5 \%$ & $10 \%$ & $1 \%$ & $5 \%$ & $10 \%$ & $1 \%$ & $5 \%$ & $10 \%$ \\
\hline \multirow[t]{5}{*}{$\widehat{\mathrm{CM}}_{n}$} & 400 & 3 & 1.0 & 4.4 & 9.1 & 5.7 & 17.2 & 25.1 & 20.6 & 43.4 & 53.9 & 51.9 & 77.5 & 85.0 & 83.9 & 94.4 & 97.1 \\
\hline & & 6 & 2.4 & 7.9 & 12.7 & 4.9 & 15.6 & 24.0 & 21.8 & 43.3 & 55.3 & 53.8 & 76.3 & 83.5 & 82.5 & 95.2 & 97.9 \\
\hline & & 10 & 1.4 & 5.8 & 10.6 & 5.6 & 16.3 & 25.8 & 21.5 & 43.6 & 55.2 & 52.1 & 76.2 & 84.3 & 82.9 & 94.3 & 96.9 \\
\hline & & 20 & 2.9 & 8.6 & 15.9 & 5.2 & 14.0 & 22.6 & 26.4 & 46.6 & 57.5 & 58.7 & 78.9 & 86.7 & 82.2 & 93.7 & 97.1 \\
\hline & & 40 & 3.6 & 10.4 & 16.7 & 9.4 & 18.3 & 25.9 & 26.9 & 44.9 & 57.7 & 61.0 & 76.4 & 86.2 & 85.8 & 95.1 & 97.9 \\
\hline \multirow[t]{2}{*}{$T_{n}$} & & 16 & 1.1 & 3.2 & 5.9 & 4.9 & 7.7 & 10.7 & 19.6 & 30.0 & 35.8 & 48.2 & 61.9 & 68.0 & 76.2 & 85.5 & 89.2 \\
\hline & & 17 & 1.1 & 3.5 & 5.2 & 3.0 & 7.9 & 10.7 & 19.1 & 28.5 & 33.3 & 46.2 & 58.7 & 65.1 & 75.8 & 84.6 & 88.7 \\
\hline \multirow[t]{5}{*}{$\widetilde{\mathrm{CM}}_{n}$} & 1000 & 3 & 1.0 & 5.0 & 8.9 & 12.8 & 30.1 & 41.4 & 60.9 & 81.3 & 88.1 & 94.6 & 99.4 & 99.7 & 100 & 100 & 100 \\
\hline & & 7 & 0.8 & 5.5 & 10.9 & 13.2 & 31.7 & 44.2 & 58.5 & 80.6 & 88.0 & 94.7 & 98.5 & 99.3 & 100 & 100 & 100 \\
\hline & & 15 & 1.2 & 6.7 & 12.0 & 14.3 & 29.4 & 39.2 & 61.5 & 81.5 & 88.7 & 95.2 & 98.9 & 99.5 & 99.8 & 100 & 100 \\
\hline & & 31 & 2.3 & 7.3 & 11.8 & 15.1 & 30.5 & 42.6 & 62.2 & 81.7 & 89.2 & 94.8 & 98.6 & 99.6 & 99.7 & 99.9 & 99.9 \\
\hline & & 63 & 3.3 & 8.2 & 13.3 & 20.1 & 34.9 & 45.1 & 63.7 & 81.9 & 89.6 & 94.7 & 98.1 & 99.3 & 99.7 & 100 & 100 \\
\hline \multirow[t]{2}{*}{$T_{n}$} & & 29 & 1.4 & 4.5 & 6.2 & 7.7 & 14.2 & 19.3 & 42.1 & 54.5 & 63.5 & 88.9 & 93.1 & 95.2 & 99.2 & 99.6 & 99.7 \\
\hline & & 30 & 1.5 & 4.2 & 6.9 & 8.4 & 15.1 & 19.8 & 43.7 & 57.1 & 64.9 & 87.6 & 93.0 & 95.3 & 99.2 & 99.7 & 99.8 \\
\hline
\end{tabular}


(a) Model (12), $n=400$

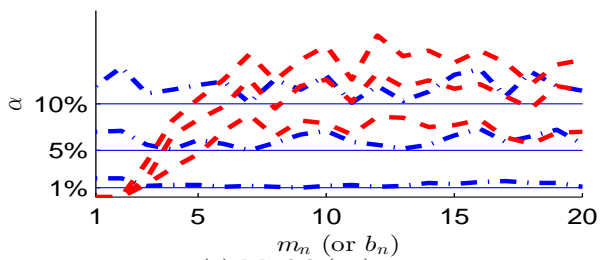

(c) Model (13), $n=400$

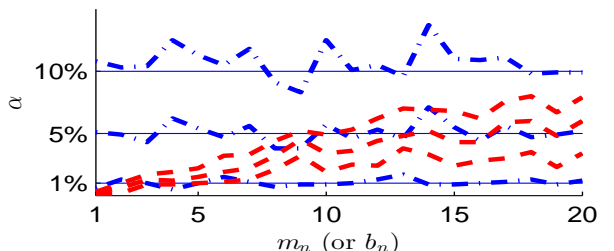

(e) Model $(14), n=400$

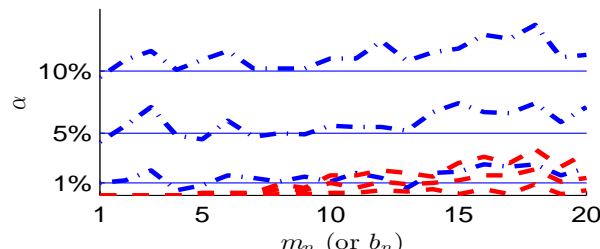

$m_{n}\left(\right.$ or $\left.b_{n}\right)$
(g) Model $(15), n=400$

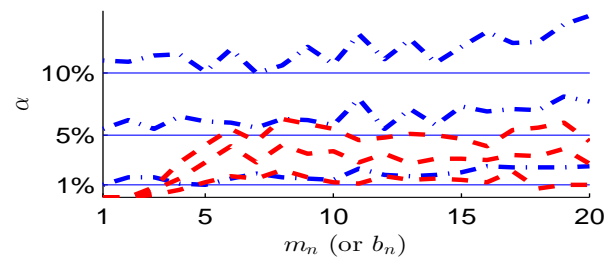

(b) Model (12), $n=1000$

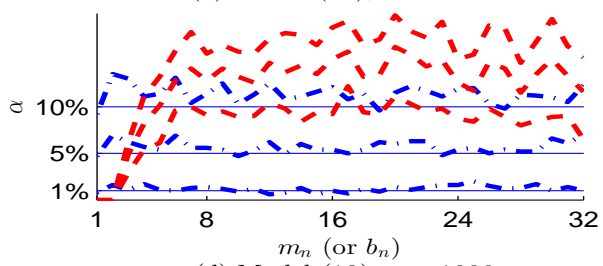

(d) Model (13), $n=1000$

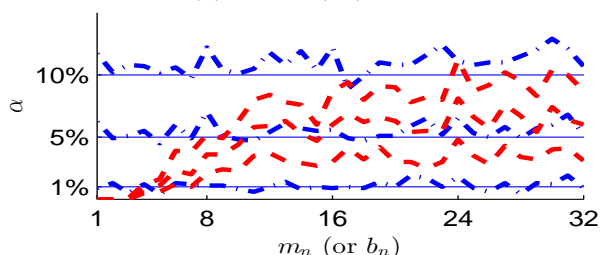

(f) Model (14), $n=1000$

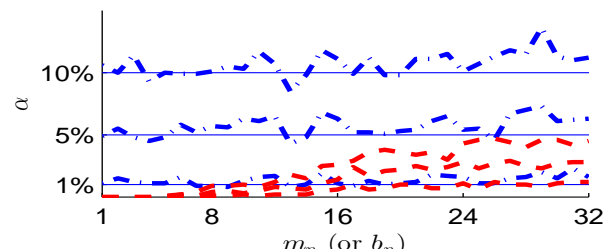

$m_{n}\left(\right.$ or $\left.b_{n}\right)$
(h) $\operatorname{Model}(15), n=1000$

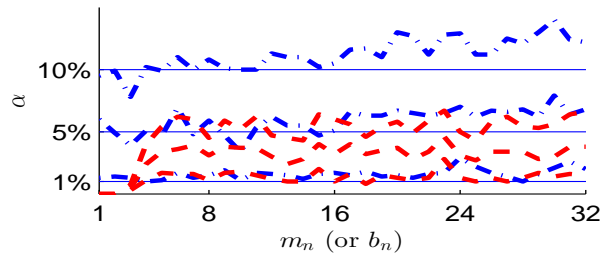

Fig. 1. In each panel, the dash (or dash-dot) lines from top to bottom are the sizes of $T_{n}$ (or $\widetilde{\mathrm{CM}}_{n}$ ) at the significance level $\alpha=10 \%, 5 \%$ and $1 \%$ for each model with $\kappa=0.0$, based on different values of $m_{n}$ (or $b_{n}$ ), where the solid lines from top to bottom are the nominal significance level $\alpha=10 \%, 5 \%$ and $1 \%$.

From Tables 1-4, we find that the sizes of $\widetilde{\mathrm{CM}}_{n}$ are close to their nominal ones when $b_{n}$ is small (e.g., $b_{n}=n^{1 / 5}$ or $2 n^{1 / 5}$ ). When $b_{n}$ gets large, $\widehat{\mathrm{CM}}_{n}$ tends to be oversized in general, but the size distortion becomes weaker as $n$ increases. This finding is consistent with the one in Shao (2011a). For $T_{n}$, we find that its size performance is very sensitive to the choice of $m_{n}$ in models (12) and (13). A visual understanding of this phenomenon can be obtained in Figure 1(a)-(d), where we plot all the empirical sizes of $T_{n}$ for different choices of $m_{n}$. As a comparison, the empirical sizes of $\widetilde{\mathrm{CM}}_{n}$ for different choices of $b_{n}$ are also plotted in Figure 1(a)-(d). It is clear that when $m_{n}$ is larger, the sizes of $T_{n}$ are seriously distorted at each significance level $\alpha$, and when $m_{n}$ is small, $T_{n}$ tends to be seriously undersized at significance levels $\alpha=5 \%$ and $10 \%$. This drawback of $T_{n}$ is unchanged even when $n$ becomes larger. By using other kernels (e.g., the Bartlett kernel and the quadratic spectral kernel), similar result holds for $T_{n}$, and hence they are not reported. Compared to $T_{n}$, the sizes of $\widetilde{\mathrm{CM}}_{n}$ are much more robust at each significance level especially when $b_{n}$ is small.

Furthermore, it is worth noting that unlike models (12) and (13), $T_{n}$ is always undersized for different choices of $m_{n}$ in models (14)-(15). This problem becomes extremely serious when $m_{n}$ 
is small. However, like models (12) and (13), the size performance of $\widetilde{\mathrm{CM}}_{n}$ is much more robust in those cases; see also Figure 1(e)-(h) for more visual evidence. From Figure 1, we find that the size performance of $\widetilde{\mathrm{CM}}_{n}$ is more satisfactory when $b_{n}=1$ or $b_{n} \approx 1$. This is probably because our $\widetilde{\mathrm{CM}}_{n}$ test applies the random weight $w_{t}^{*}$ to $\widetilde{\varepsilon}_{t} \widetilde{\varepsilon}_{t+h}$ not to $\widetilde{\varepsilon}_{t}$, and the dependence structure of $\widetilde{\varepsilon}_{t} \widetilde{\varepsilon}_{t+h}$ for $h \geq 1$ in models (12)-(15) is very weak (e.g., the autocovariance of $\varepsilon_{t} \varepsilon_{t+h}$ at each lag $j(>0)$ is zero in models (12)-(13)). This feature of our $\widetilde{\mathrm{CM}}_{n}$ test may help us to explain its robust size performance, and a rigorous justification on this conjecture is an interesting topic for future study. Overall, we know that the sizes of $\widetilde{\mathrm{CM}}_{n}$ are precise especially when $b_{n}$ is small, while the sizes of $T_{n}$ could be seriously undersized or oversized in most cases of $m_{n}$. It means that the performance of $T_{n}$ is heavily relied on whether we can obtain an optimal $m_{n}$, but this is not the case for $\widetilde{\mathrm{CM}}_{n}$. Considering the difficulty of selecting the optimal bandwidth in most of the nonparametric methods for practitioners, $\widehat{\mathrm{CM}}_{n}$ has a size advantage over $T_{n}$ in this direction.

Next, we consider the power performances for $\widetilde{\mathrm{CM}}_{n}$ and $T_{n}$, and the conclusion is generally as expected. First, all the powers become large as $n$ increases. Second, $\widetilde{\mathrm{CM}}_{n}$ is generally more powerful than $T_{n}$ for all examined alternatives in models (14)-(15), while $T_{n}$ has a power advantage over $\widetilde{\mathrm{CM}}_{n}$ in model (12) and model (13) with a large $\kappa$. The power advantage of $T_{n}$ over $\widetilde{C M}_{n}$ in model (12) is probably because the residuals of a fitted MA(1) model exhibit certain autocorrelations at some large lags. The power advantage of $T_{n}$ over $\widetilde{\mathrm{CM}}_{n}$ in model (13) is quite reasonable, since the sample autocovariance of the residuals from the fitted $\operatorname{AR}(1)$ model becomes more significantly different from zero when $\kappa$ is larger. Overall, although $\widehat{\mathrm{CM}}_{n}$ does not have a consistent power advantage over $T_{n}$, it is reasonable to recommend $\widetilde{\mathrm{CM}}_{n}$ in practice since it has a very robust size performance especially when the block size is small.

\section{APPLiCATION TO S\&P 500 STOCK INDEX}

In this section, we revisit the real example on S\&P 500 stock index in Escanciano and Velasco (2006). We consider two sample periods for the S\&P 500 stock index. The first period is from 3 January 1994 until 31 December 1997 with a total of 1011 observations. The second period is from 2 January 1998 until 28 August 2002 with a total of 1170 observations. Denote the logreturn of both series (after mean-adjusted) by $y_{1 t}$ and $y_{2 t}$, respectively. The generalized spectral tests in Escanciano and Velasco (2006, p.172) indicate that $y_{1 t}$ is non-MDS at the significance level $\alpha=5 \%$, while $y_{2 t}$ is non-MDS at the significance level $\alpha=10 \%$. Thus, we are of interest to test whether $y_{1 t}$ or $y_{2 t}$ is a weak white noise (i.e., an uncorrelated sequence) by using $\widetilde{\mathrm{CM}}_{n}$. As in Section 4 , we choose $b_{n}=n^{1 / 5}, 2 n^{1 / 5}, \sqrt{n} / 2, \sqrt{n}$ or $2 \sqrt{n}$, and it delivers $b_{n}=3,7,15,31$ for $y_{1 t}$ and 4, 8, 16,32 for $y_{2 t}$. The corresponding results for $\widetilde{\mathrm{CM}}_{n}$ are listed in Table 5, from which we can not reject the hypothesis that $y_{1 t}$ or $y_{2 t}$ is a weak white noise at the $5 \%$ significance level, and this conclusion is unchanged for all choices of $b_{n}$. Thus, a weak but non-MDS process should be suitable to fit $y_{1 t}$ or $y_{2 t}$.

Next, we use $\widetilde{\mathrm{CM}}_{n}$ to check whether a weak MA(3) model defined as $y_{t}=\varepsilon_{t}+\varphi \varepsilon_{t-3}$ for $|\varphi|<1$, is adequate to fit $y_{1 t}$ or $y_{2 t}$. Based on LS estimation, the fitted weak MA(3) models for $y_{1 t}$ and $y_{2 t}$ are as follows:

$$
\begin{aligned}
& y_{1 t}=\varepsilon_{1 t}-0.0482 \varepsilon_{1 t-3}, \\
& y_{2 t}=\varepsilon_{2 t}-0.0423 \varepsilon_{2 t-3},
\end{aligned}
$$


Table 5. p-values of $\widetilde{C M}_{n}$ for testing the adequacy of a weak white noise on two $S \& P 500$ stock indexes

\begin{tabular}{lllllll}
\hline \hline & & \multicolumn{5}{c}{$b_{n}$} \\
\cline { 3 - 7 } Series & $n^{1 / 5}$ & $2 n^{1 / 5}$ & $\sqrt{n} / 2$ & $\sqrt{n}$ & $2 \sqrt{n}$ \\
$y_{1 t}$ & p-value $^{\dagger}$ & 0.6900 & 0.6537 & 0.5050 & 0.6257 & 0.5637 \\
$y_{2 t}$ & p-value & 0.5110 & 0.5180 & 0.4017 & 0.4157 & 0.2783 \\
\hline${ }^{\dagger} p$-values bootstrapped by the BRW method with $J=3000$. &
\end{tabular}

where the estimated values of $\sigma_{\varepsilon_{1}}^{2}=6.2 \times 10^{-5}$ and $\sigma_{\varepsilon_{2}}^{2}=1.8 \times 10^{-4}$. The p-values of $\widetilde{\mathrm{CM}}_{n}$ in Table 6 indicate that models (16)-(17) are adequate at the 5\% significance level, while the $p$ values of the Ljung-Box test statistics $Q(M)$ and Li-Mak test statistics $Q^{2}(M)$ in Table 7 imply that models (16)-(17) are not strong at the same significance level. Note that a Bilinear model like (15) with $\kappa=0$ has a weak MA(3) representation. Thus, it motivates us to fit $y_{1 t}$ or $y_{2 t}$ by the following Bilinear-GARCH model:

$$
\left\{\begin{array}{l}
y_{t}=\eta_{t}+u y_{t-1} \eta_{t-2} \\
\eta_{t}=\sqrt{h_{t}} \nu_{t} \text { and } h_{t}=\omega+\alpha \eta_{t-1}^{2}+\beta h_{t-1}
\end{array}\right.
$$

where $|u|\langle 1, \omega\rangle 0, \alpha, \beta \geq 0$ and $\nu_{t}$ is an iid re-scaled error sequence. For each series, model (18) is estimated by using the QMLE method (see, e.g., Ling (2007) and Francq and Zakoïan (2010)). The related results are summarized in Table 8, from which we know that model (18) is adequate to fit $y_{2 t}$, while a marginal autocorrelation up to lag 6 is detected in the fitted conditional mean model for $y_{1 t}$. Based on this, we re-fit $y_{1 t}$ by another Bilinear-GARCH model:

$$
\left\{\begin{array}{l}
y_{t}=v \eta_{t-1}+\eta_{t}+u y_{t-1} \eta_{t-2} \\
\eta_{t}=\sqrt{h_{t}} \nu_{t} \text { and } h_{t}=\omega+\alpha \eta_{t-1}^{2}+\beta h_{t-1}
\end{array}\right.
$$

where $|v|<1,|u|<1, \omega>0, \alpha, \beta \geq 0$ and $\nu_{t}$ is an iid re-scaled error sequence. The related results for the fitted model (19) are given in Table 8, from which we know that model (19) is adequate in fitting $y_{1 t}$.

Table 6. p-values of $\widetilde{C M}_{n}$ for testing the adequacy of a weak MA(3) model on two S\&P 500 stock indexes

\begin{tabular}{ccccccc}
\hline \hline & & \multicolumn{5}{c}{$b_{n}$} \\
\cline { 3 - 7 } Series & $n^{1 / 5}$ & $2 n^{1 / 5}$ & $\sqrt{n} / 2$ & $\sqrt{n}$ & $2 \sqrt{n}$ \\
& & & & & & \\
$y_{1 t}$ & p-value $^{\dagger}$ & 0.9087 & 0.8923 & 0.8637 & 0.9707 & 0.9627 \\
$y_{2 t}$ & p-value & 0.8420 & 0.8630 & 0.6720 & 0.5560 & 0.4940
\end{tabular}

${ }^{\dagger} p$-values bootstrapped by the BRW method with $J=3000$. 
Table 7. $p$-values of $Q(M)$ and $Q^{2}(M)$ for testing the adequacy of a strong MA(3) model on two $S \& P 500$ stock indexes

\begin{tabular}{cccccccc}
\hline \hline Series & & $Q(6)$ & $Q(12)$ & $Q(24)$ & $Q^{2}(6)$ & $Q^{2}(12)$ & $Q^{2}(24)$ \\
& & & & & & & \\
$y_{1 t}$ & $\mathrm{p}$-value & 0.3453 & 0.0106 & 0.0588 & 0.0000 & 0.0000 & 0.0000 \\
& & & & & & & \\
$y_{2 t}$ & $\mathrm{p}$-value & 0.2756 & 0.1774 & 0.2689 & 0.0000 & 0.0000 & 0.0000 \\
& & & & & & & \\
\hline
\end{tabular}

Table 8. QMLE-fitted model and its corresponding portmanteau tests on two S\&P 500 stock indexes

\begin{tabular}{|c|c|c|c|c|c|c|c|c|c|c|c|}
\hline & & & & $Q M L E$ & & & & & & & \\
\hline \multirow{3}{*}{ Model (18) } & Series & $v_{n}$ & $u_{n}$ & $\omega_{n}$ & $\alpha_{n}$ & $\beta_{n}$ & $\sigma_{\nu}^{2}$ & $Q(6)$ & $Q(24)$ & $Q^{2}(6)$ & $Q^{2}(24)^{\dagger}$ \\
\hline & $y_{1 t}$ & --- & 0.9961 & 0.0000 & 0.1045 & 0.8686 & 0.9984 & 0.0461 & 0.2591 & 0.9517 & 0.9945 \\
\hline & $y_{2 t}$ & --- & 0.8004 & 0.0000 & 0.1129 & 0.8213 & 0.9984 & 0.4106 & 0.3525 & 0.2549 & 0.6193 \\
\hline Model (19) & $y_{1 t}$ & 0.0703 & 0.8001 & 0.0000 & 0.1083 & 0.8650 & 0.9971 & 0.4310 & 0.6353 & 0.9614 & 0.9951 \\
\hline
\end{tabular}

\section{CONCLUding REMARKS}

In this paper, we study the asymptotic property of a CM-type spectral test statistic $\widetilde{\mathrm{CM}}_{n}$ for checking the adequacy of an ARMA model with uncorrelated errors. By releasing the martingale difference assumption on the error terms, $\widetilde{\mathrm{CM}}_{n}$ is applicable to a large class of uncorrelated nonlinear processes. Since we do not specify the form of error terms, the limiting distribution of $\widetilde{\mathrm{CM}}_{n}$ is not pivotal, and so a novel BRW method is necessary to bootstrap the critical values of $\widetilde{\mathrm{CM}}_{n}$. Simulation studies show that the size and power performances of $\widetilde{\mathrm{CM}}_{n}$ are robust to the selection of block size $b_{n}$ in BRW method especially when the sample size is large, while the size of kernel-based test $T_{n}$ in Shao (2011b) is always sensitive to the choice of the bandwidth $m_{n}$. In addition, $\widetilde{\mathrm{CM}}_{n}$ has a power advantage over $T_{n}$ under most of the examined alternatives. By revisiting two S\&P 500 stock index series in Escanciano and Velasco (2006), $\widetilde{\mathrm{CM}}_{n}$ suggests that the Bilinear-GARCH models are adequate to fit both series. This empirical example illustrates that although some economic or financial series is not a martingale difference sequence, it is still very likely to be an uncorrelated sequence. Our test statistic $\widetilde{\mathrm{CM}}_{n}$ now gives us a way to check for the adequacy of ARMA models driven by an uncorrelated error sequence. Moreover, once a weak ARMA model is found to be adequate in fitting the given series, some non-linear processes with a weak ARMA representation may also be considered to fit this series adequately. This point of view should be important for practitioners.

\section{ACKNOWLEDGEMENT}

The authors greatly appreciate the very constructive suggestions and comments of P. Doukhan, three anonymous referees, the Associate Editor, and the Editor J. Fan that greatly improve this article. This work is supported by Research Grants Council of the Hong Kong SAR Government, GRF grant HKU703711P, National Natural Science Foundation of China (No.11201459), Pres- 
ident Fund of Academy of Mathematics and Systems Science, CAS (No.2014-cjrwlzx-zk), and Key Laboratory of RCSDS, CAS.

Denote by $W_{h}(j)=\int_{0}^{\pi} h(\lambda) \psi_{j}(\lambda) d \lambda$ for any $h \in L_{2}[0, \pi]$; by $P_{j}=\int_{0}^{\pi} \psi_{j}^{2}(\lambda) d \lambda$ for $j \in \mathcal{N}$; by $C$ a positive generic constant which may vary from place to place. Note that $P_{j} \leq C j^{-2}$ uniformly in $j \in \mathcal{N}$, and $\int_{0}^{\pi} \psi_{j}(\lambda) \psi_{k}(\lambda) d \lambda=0$ when $j \neq k$ and $j, k \in \mathcal{N}$. In order to prove Theorem 1, we rewrite

$$
\begin{aligned}
\widetilde{S}_{n}(\lambda) & =\left[\widetilde{S}_{n}(\lambda)-\breve{S}_{n}(\lambda)\right]+\breve{S}_{n}(\lambda) \\
& =\left[\widetilde{S}_{n}(\lambda)-\breve{S}_{n}(\lambda)\right]+\left[\breve{S}_{n}(\lambda)-\breve{S}_{n}(\lambda)\right]+\breve{S}_{n}(\lambda) \\
& =I_{1 n}(\lambda)+I_{2 n}(\lambda)+\breve{S}_{n}(\lambda) \text { say. }
\end{aligned}
$$

where $\breve{S}_{n}(\lambda)=\sum_{j=1}^{n-1} \sqrt{n} \breve{\gamma}(j) \psi_{j}(\lambda)$ with $\breve{\gamma}(j)=n^{-1} \sum_{t=1+|j|}^{n} \breve{\varepsilon}_{t} \breve{\varepsilon}_{t-|j|}$ and $\breve{\varepsilon}_{t}=\varepsilon_{t}\left(\theta_{n}\right)$. Then, we need the following five lemmas:

Lemma A1. Suppose that Assumption 1 holds. Then, there exist $C>0$ and $\rho \in(0,1)$ such that

$$
\begin{aligned}
& \text { (i) } \sup _{\Theta}\left|\varepsilon_{t}(\theta)\right|<C \xi_{\rho t}, \sup _{\Theta}\left\|\frac{\partial \varepsilon_{t}(\theta)}{\partial \theta}\right\|<C \xi_{\rho t-1}, \text { and } \sup _{\Theta}\left\|\frac{\partial^{2} \varepsilon_{t}(\theta)}{\partial \theta \partial \theta^{\prime}}\right\|<C \xi_{\rho t-1} ; \\
& \text { (ii) } \sup _{\Theta}\left|\varepsilon_{t}(\theta)-\widetilde{\varepsilon}_{t}(\theta)\right| \leq O\left(\rho^{t}\right) \xi_{\rho 0}, \sup _{\Theta}\left\|\frac{\partial \varepsilon_{t}(\theta)}{\partial \theta}-\frac{\partial \widetilde{\varepsilon}_{t}(\theta)}{\partial \theta}\right\| \leq O\left(\rho^{t}\right) \xi_{\rho 0}, \\
& \text { and } \sup _{\Theta}\left\|\frac{\partial^{2} \varepsilon_{t}(\theta)}{\partial \theta \partial \theta^{\prime}}-\frac{\partial^{2} \widetilde{\varepsilon}_{t}(\theta)}{\partial \theta \partial \theta^{\prime}}\right\| \leq O\left(\rho^{t}\right) \xi_{\rho 0},
\end{aligned}
$$

where $\xi_{\rho t}=1+\sum_{i=0}^{\infty} \rho^{i}\left|y_{t-i}\right|$.

Proof. The proof follows directly from Lemmas A.1 and A.4 in Ling (2007).

Lemma A2. Suppose that Assumptions 1-2 hold. Then, $\left\|I_{1 n}(\lambda)\right\|^{2}=o_{p}(1)$.

Proof. By direct calculation, we have

$$
E\left\|I_{1 n}(\lambda)\right\|^{2}=\frac{1}{n} \sum_{j=1}^{n-1} E\left(\sum_{t=1+j}^{n} b_{t j}\left(\theta_{n}\right)\right)^{2} P_{j},
$$

where $b_{t j}(\theta)=\varepsilon_{t}(\theta) \varepsilon_{t-j}(\theta)-\widetilde{\varepsilon}_{t}(\theta) \widetilde{\varepsilon}_{t-j}(\theta)$. By Minkowski inequality, it follows that

$$
\begin{aligned}
E\left\|I_{1 n}(\lambda)\right\|^{2} & \leq \frac{1}{n} \sum_{j=1}^{n-1}\left(\sum_{t=1+j}^{n}\left\{E\left[b_{t j}\left(\theta_{n}\right)\right]^{2}\right\}^{1 / 2}\right)^{2} P_{j} \\
& \leq \frac{1}{n} \sum_{j=1}^{n-1}\left(\sum_{t=1+j}^{n}\left\{E\left[\sup _{\Theta}\left\|b_{t j}(\theta)\right\|\right]^{2}\right\}^{1 / 2}\right)^{2} P_{j} .
\end{aligned}
$$

By Lemma A1, we know that there exists a constant $\rho \in(0,1)$ such that

$$
\begin{aligned}
\sup _{\Theta}\left\|b_{t j}(\theta)\right\| & \leq \sup _{\Theta}\left\|\left[\varepsilon_{t}(\theta)-\widetilde{\varepsilon}_{t}(\theta)\right] \varepsilon_{t-j}(\theta)\right\|+\sup _{\Theta}\left\|\widetilde{\varepsilon}_{t}(\theta)\left[\varepsilon_{t-j}(\theta)-\widetilde{\varepsilon}_{t-j}(\theta)\right]\right\| \\
& \leq O\left(\rho^{t}\right) \xi_{\rho 0} \xi_{\rho t-j}+O\left(\rho^{t-j}\right) \xi_{\rho 0} \xi_{\rho t} .
\end{aligned}
$$


Note that $E\left|\xi_{\rho t}\right|^{4}<\infty$ by Assumption 2. Thus, from (A2), by Hölder inequality, we can show that

$$
\begin{aligned}
E\left\|I_{1 n}(\lambda)\right\|^{2} \leq & \frac{1}{n} \sum_{j=1}^{n-1}\left(\sum_{t=1+j}^{n}\left\{O\left(\rho^{2 t}\right) E\left[\xi_{\rho 0} \xi_{\rho t-j}\right]^{2}+O\left(\rho^{2(t-j)}\right) E\left[\xi_{\rho 0} \xi_{\rho t}\right]^{2}\right\}^{1 / 2}\right)^{2} P_{j} \\
\leq & \frac{1}{n} \sum_{j=1}^{n-1}\left(\sum _ { t = 1 + j } ^ { n } \left\{O\left(\rho^{2 t}\right)\left(E\left[\xi_{\rho 0}\right]^{4} E\left[\xi_{\rho t-j}\right]^{4}\right)^{1 / 2}\right.\right. \\
& \left.\left.+O\left(\rho^{2(t-j)}\right)\left(E\left[\xi_{\rho 0}\right]^{4} E\left[\xi_{\rho t}\right]^{4}\right)^{1 / 2}\right\}^{1 / 2}\right)^{2} P_{j} \\
\leq & \frac{1}{n} \sum_{j=1}^{n-1}\left(\sum_{t=1+j}^{n}\left\{O\left(\rho^{t}\right)+O\left(\rho^{t-j}\right)\right\}\right)^{2} P_{j}=O\left(\frac{1}{n}\right)
\end{aligned}
$$

which implies that $\left\|I_{1 n}(\lambda)\right\|^{2}=o_{p}(1)$.

Lemma A3. Suppose that Assumptions 1-3 hold. Then,

(i) $E\left[\frac{\partial l_{t}\left(\breve{\theta}_{0}\right)}{\partial \theta}\right]=0$

(ii) $\sqrt{n}\left(\theta_{n}-\check{\theta}_{0}\right)=O_{p}(1)$ with $\sqrt{n}\left(\theta_{n}-\check{\theta}_{0}\right)=-\Sigma^{-1}\left[\frac{1}{\sqrt{n}} \sum_{t=1}^{n} \frac{\partial l_{t}\left(\check{\theta}_{0}\right)}{\partial \theta}\right]+o_{p}(1)$,

where $l_{t}(\theta)$ is defined as in Assumption 3(ii).

Proof. (i) By Lemma A1, it is not hard to show that

$$
\begin{aligned}
& \sup _{\Theta}\left\|\frac{1}{\sqrt{n}} \sum_{t=1}^{n}\left[\frac{\partial l_{t}(\theta)}{\partial \theta}-\frac{\partial \widetilde{l}_{t}(\theta)}{\partial \theta}\right]\right\|=o_{p}(1), \\
& \sup _{\Theta}\left\|\frac{1}{n} \sum_{t=1}^{n}\left[\frac{\partial^{2} l_{t}(\theta)}{\partial \theta \partial \theta^{\prime}}-\frac{\partial^{2} \widetilde{l}_{t}(\theta)}{\partial \theta \partial \theta^{\prime}}\right]\right\|=o_{p}(1) .
\end{aligned}
$$

Then, since $\partial \widetilde{l}_{t}\left(\theta_{n}\right) / \partial \theta=0$, by Taylor's expansion and (A3)-(A4), we have

$$
\begin{aligned}
\theta_{n}-\breve{\theta}_{0} & =-\left[\frac{1}{n} \sum_{t=1}^{n} \frac{\partial^{2} \widetilde{l}_{t}\left(\zeta_{n}\right)}{\partial \theta \partial \theta^{\prime}}\right]^{-1}\left[\frac{1}{n} \sum_{t=1}^{n} \frac{\partial \widetilde{l}_{t}\left(\breve{\theta}_{0}\right)}{\partial \theta}\right] \\
& =-\left[\frac{1}{n} \sum_{t=1}^{n} \frac{\partial^{2} l_{t}\left(\zeta_{n}\right)}{\partial \theta \partial \theta^{\prime}}\right]^{-1}\left[\frac{1}{n} \sum_{t=1}^{n} \frac{\partial l_{t}\left(\breve{\theta}_{0}\right)}{\partial \theta}\right]+o_{p}(1)
\end{aligned}
$$

where $\zeta_{n}$ lies between $\theta_{n}$ and $\check{\theta}_{0}$. By Lemma A1(i) and Assumption 2, we know that

$$
E \sup _{\Theta}\left\|\frac{\partial^{2} l_{t}(\theta)}{\partial \theta \partial \theta^{\prime}}\right\| \leq C E\left(\xi_{\rho t-1}^{2}+\xi_{\rho t} \xi_{\rho t-1}\right)<\infty
$$

for some $\rho \in(0,1)$. Thus, by Theorem 3.1 in Ling and McAleer (2003), we have

$$
\frac{1}{n} \sum_{t=1}^{n} \frac{\partial^{2} l_{t}\left(\zeta_{n}\right)}{\partial \theta \partial \theta^{\prime}}=E\left[\frac{\partial^{2} l_{t}\left(\zeta_{n}\right)}{\partial \theta \partial \theta^{\prime}}\right]+o_{p}(1)=\Sigma+o_{p}(1)
$$


where the last equality holds by the dominated convergence theorem and the fact that $\xi_{n} \rightarrow_{p} \breve{\theta}_{0}$ as $n \rightarrow \infty$ by Assumption 3. By (A5)-(A6) and the ergodic theorem, it follows that

$$
\theta_{n}-\check{\theta}_{0}=-\Sigma^{-1}\left[\frac{1}{n} \sum_{t=1}^{n} \frac{\partial l_{t}\left(\check{\theta}_{0}\right)}{\partial \theta}\right]+o_{p}(1)=-\Sigma^{-1} E\left[\frac{\partial l_{t}\left(\check{\theta}_{0}\right)}{\partial \theta}\right]+o_{p}(1) .
$$

Since $\theta_{n}-\check{\theta}_{0}=o_{p}(1)$ by Assumption 3 , it implies that (i) holds.

(ii) By (A3)-(A5), it is not hard to see that

$$
\sqrt{n}\left(\theta_{n}-\check{\theta}_{0}\right)=-\left[\frac{1}{n} \sum_{t=1}^{n} \frac{\partial^{2} l_{t}\left(\zeta_{n}\right)}{\partial \theta \partial \theta^{\prime}}\right]^{-1}\left[\frac{1}{\sqrt{n}} \sum_{t=1}^{n} \frac{\partial l_{t}\left(\check{\theta}_{0}\right)}{\partial \theta}\right]+o_{p}(1) .
$$

Note that $\partial l_{t}\left(\breve{\theta}_{0}\right) / \partial \theta=2 \check{\varepsilon}_{t}\left(\partial \check{\varepsilon}_{t} / \partial \theta\right)$. Thus, by Assumptions 1 and 2(i), Lemmas 3-4 in Francq and Zakoïan (1998) implies that $n^{-1 / 2} \sum_{t=1}^{n} \partial l_{t}\left(\check{\theta}_{0}\right) / \partial \theta=O_{p}(1)$. By (A6), it follows that (ii) holds.

Lemma A4. Suppose that Assumptions 1-3 hold. Then,

$$
\left\|I_{2 n}(\lambda)-A_{n}^{\prime}(\lambda)\left[\sqrt{n}\left(\theta_{n}-\breve{\theta}_{0}\right)\right]\right\|^{2}=o_{p}(1),
$$

where

$$
A_{n}(\lambda)=\sum_{j=1}^{n-1} E\left[\frac{\partial\left(\check{\varepsilon}_{t} \check{\varepsilon}_{t-j}\right)}{\partial \theta}\right] \psi_{j}(\lambda) .
$$

Proof. By Taylor's expansion, we have $\widehat{\varepsilon}_{t}-\check{\varepsilon}_{t}=\left(\partial \varepsilon_{t}\left(\zeta_{n}\right) / \partial \theta^{\prime}\right)\left(\theta_{n}-\check{\theta}_{0}\right)$, where $\zeta_{n}$ lies between $\theta_{n}$ and $\breve{\theta}_{0}$. Then, it follows that

$$
I_{2 n}(\lambda)=\sum_{j=1}^{n-1}\left\{\frac{1}{n} \sum_{t=1+j}^{n}\left[\frac{\partial \varepsilon_{t}\left(\zeta_{n}\right)}{\partial \theta^{\prime}} \widehat{\varepsilon}_{t-j}+\check{\varepsilon}_{t} \frac{\partial \varepsilon_{t-j}\left(\zeta_{n}\right)}{\partial \theta^{\prime}}\right] \psi_{j}(\lambda)\right\}\left[\sqrt{n}\left(\theta_{n}-\breve{\theta}_{0}\right)\right],
$$

which entails

$$
I_{2 n}(\lambda)=\left\{I_{2 n}^{(1)}\left(\lambda, \zeta_{n}, \theta_{n}\right)+I_{2 n}^{(2)}\left(\lambda, \zeta_{n}\right)+I_{2 n}^{(3)}(\lambda)\right\}\left[\sqrt{n}\left(\theta_{n}-\breve{\theta}_{0}\right)\right]
$$

where

$$
\begin{aligned}
I_{2 n}^{(1)}\left(\lambda, \theta_{1}, \theta_{2}\right) & =\sum_{j=1}^{n-1}\left\{\frac{1}{n} \sum_{t=1+j}^{n}\left[\frac{\partial \varepsilon_{t}\left(\theta_{1}\right)}{\partial \theta^{\prime}} \varepsilon_{t-j}\left(\theta_{2}\right)-E\left(\frac{\partial \check{\varepsilon}_{t}}{\partial \theta^{\prime}} \check{\varepsilon}_{t-j}\right)\right] \psi_{j}(\lambda)\right\}, \\
I_{2 n}^{(2)}\left(\lambda, \theta_{1}\right) & =\sum_{j=1}^{n-1}\left\{\frac{1}{n} \sum_{t=1+j}^{n}\left[\check{\varepsilon}_{t} \frac{\partial \varepsilon_{t-j}\left(\theta_{1}\right)}{\partial \theta^{\prime}}-E\left(\check{\varepsilon}_{t} \frac{\partial \check{\varepsilon}_{t-j}}{\partial \theta^{\prime}}\right)\right] \psi_{j}(\lambda)\right\}, \\
I_{2 n}^{(3)}(\lambda) & =\sum_{j=1}^{n-1}\left\{\frac{n-j}{n}\left[E\left(\frac{\partial \check{\varepsilon}_{t}}{\partial \theta^{\prime}} \check{\varepsilon}_{t-j}\right)+E\left(\check{\varepsilon}_{t} \frac{\partial \check{\varepsilon}_{t-j}}{\partial \theta^{\prime}}\right)\right] \psi_{j}(\lambda)\right\} .
\end{aligned}
$$

We first consider $I_{2 n}^{(1)}\left(\lambda, \zeta_{n}, \theta_{n}\right)$. By direct calculation, we have

$$
E\left\|I_{2 n}^{(1)}\left(\lambda, \zeta_{n}, \theta_{n}\right)\right\|^{2}=\sum_{j=1}^{n-1}\left(E c_{n j}^{2}\right) P_{j},
$$

where

$$
c_{n j}=\frac{1}{n} \sum_{t=1+j}^{n}\left[\frac{\partial \varepsilon_{t}\left(\zeta_{n}\right)}{\partial \theta^{\prime}} \varepsilon_{t-j}\left(\theta_{n}\right)-E\left(\frac{\partial \check{\varepsilon}_{t}}{\partial \theta^{\prime}} \check{\varepsilon}_{t-j}\right)\right] .
$$


As for (A6), by Assumptions 2 and 3(i) and Lemma A1(i), we can show that uniformly in $j \in\{1, \cdots, n-\quad 475$ $1\}, E c_{n j}^{2}=o(1)$. Thus, since $\sum_{j=1}^{\infty} P_{j}<\infty$, by (A8), it is straightforward to see that

$$
E\left\|I_{2 n}^{(1)}\left(\lambda, \zeta_{n}, \theta_{n}\right)\right\|^{2}=\sum_{j=1}^{n-1} o\left(P_{j}\right)=o(1),
$$

which implies that $\left\|I_{2 n}^{(1)}\left(\lambda, \zeta_{n}, \theta_{n}\right)\right\|^{2}=o_{p}(1)$. Similarly, $\left\|I_{2 n}^{(2)}\left(\lambda, \zeta_{n}\right)\right\|^{2}=o_{p}(1)$.

Next, we consider $I_{2 n}^{(3)}(\lambda)$. By direct calculation and the fact $P_{j}=O\left(j^{-2}\right)$, we have

$$
E\left\|I_{2 n}^{(3)}(\lambda)-A_{n}(\lambda)\right\|^{2}=\sum_{j=1}^{n-1} \frac{j^{2}}{n^{2}}\left[E\left(\frac{\partial \breve{\varepsilon}_{t}}{\partial \theta^{\prime}} \breve{\varepsilon}_{t-j}\right)+E\left(\check{\varepsilon}_{t} \frac{\partial \breve{\varepsilon}_{t-j}}{\partial \theta^{\prime}}\right)\right]^{2} P_{j}=O\left(n^{-1}\right) .
$$

Now, the conclusion follows from (A7) and Lemma A3(ii).

Lemma A5. Suppose that Assumptions 1-3 hold. Then,

$$
\left\|\sum_{j=1}^{n-1}\left(\frac{1}{\sqrt{n}} \sum_{t=1}^{j} z_{t j}\right) \psi_{j}(\lambda)\right\|^{2}=o_{p}(1),
$$

where $z_{t j}$ is defined as in (3).

Proof. First, by Lemma A3(i), we have $E z_{t j}=0$. Then, as for (A6), by Assumptions 1-2, it is not hard to show that

$$
E\left[\frac{1}{j} \sum_{t=1}^{j} z_{t j}\right]^{2} \rightarrow 0 \quad \text { as } j \rightarrow \infty .
$$

Thus, $\forall \varepsilon>0$, there exists a $n_{0}(\varepsilon)$ such that when $j \geq n_{0}$,

$$
E\left[\frac{1}{j} \sum_{t=1}^{j} z_{t j}\right]^{2}<\varepsilon .
$$

Next, by direct calculation, for $n \geq \max \left(n_{0}+1,\left\lfloor\varepsilon^{-1}\right\rfloor\right)$, we have

$$
\begin{aligned}
E & \left\|\sum_{j=1}^{n-1}\left(\frac{1}{\sqrt{n}} \sum_{t=1}^{j} z_{t j}\right) \psi_{j}(\lambda)\right\|^{2} \\
& =\frac{1}{n} \sum_{j=1}^{n-1} j^{2} E\left[\frac{1}{j} \sum_{t=1}^{j} z_{t j}\right]^{2} P_{j} \\
& =\frac{1}{n} \sum_{j=1}^{n_{0}-1} j^{2} E\left[\frac{1}{j} \sum_{t=1}^{j} z_{t j}\right]^{2} P_{j}+\frac{1}{n} \sum_{j=n_{0}}^{n-1} j^{2} E\left[\frac{1}{j} \sum_{t=1}^{j} z_{t j}\right]^{2} P_{j} \\
& \leq O\left(\frac{1}{n}\right)+\frac{\varepsilon}{n} \sum_{j=n_{0}}^{n-1} j^{2} P_{j} \\
& =O\left(\frac{1}{n}\right)+O(\varepsilon)=O(\varepsilon) .
\end{aligned}
$$

Thus, it follows that the conclusion holds.

Proof of Theorem 1. By (A1) and Lemmas A2, A4 and A5, it suffices to show that $\bar{S}_{n}(\lambda)-$ $E\left\{\bar{S}_{n}(\lambda)\right\} \Rightarrow S(\lambda)$ as $n \rightarrow \infty$, where $\bar{S}_{n}(\lambda)=\sum_{j=1}^{n-1} \sqrt{n} \bar{\lambda}(j) \psi_{j}(\lambda)$ with $\bar{\lambda}_{n}(\lambda)=n^{-1} \sum_{t=1+|j|}^{n} e_{t, j}$. 
Here, we have used the fact that $E\left\{\breve{S}_{n}(\lambda)\right\}=E\left\{\bar{S}_{n}(\lambda)\right\}$ by Lemma A3(i). For each fixed integer $K \in$ $\{1, \cdots, n-1\}$, we rewrite

$$
\bar{S}_{n}(\lambda)=\sum_{j=1}^{K} \sqrt{n} \bar{\lambda}(j) \psi_{j}(\lambda)+\sum_{j=K+1}^{n-1} \sqrt{n} \bar{\lambda}(j) \psi_{j}(\lambda)=: \bar{S}_{n}^{K}(\lambda)+R_{n}^{K}(\lambda) .
$$

Then, as in Shao (2011a), the conclusion holds from the following three claims:

(a). For any $h \in L_{2}[0, \pi]$, the finite dimensional distributions of $\left\langle\bar{S}_{n}^{K}-E\left(\bar{S}_{n}^{K}\right), h\right\rangle$ converge to those of $\left\langle S^{K}(\lambda), h\right\rangle$, where $S^{K}(\lambda)$ is a Gaussian process with zero mean and asymptotic projected variances

$$
\sigma_{h, K}^{2}=\operatorname{var}\left[\left\langle S^{K}, h\right\rangle\right]=\sum_{j=1}^{K} \sum_{k=1}^{K} \sum_{d=-\infty}^{\infty} \operatorname{cov}\left(e_{t, j}, e_{t-d, k}\right) W_{h}(j) W_{h}(k) .
$$

(b). The sequence $\left\{\bar{S}_{n}^{K}(\lambda)\right\}$ is tight.

(c). For $\forall \varepsilon>0, \lim _{K \rightarrow \infty} \lim _{n \rightarrow \infty} P\left(\left\|R_{n}^{K}(\lambda)-E\left\{R_{n}^{K}(\lambda)\right\}\right\|>\varepsilon\right)=0$.

Q.E.D.

$$
\begin{aligned}
\left\langle\bar{S}_{n}^{K}-E\left(\bar{S}_{n}^{K}\right), h\right\rangle= & \frac{1}{\sqrt{n}} \sum_{j=1}^{K} \sum_{t=j+1}^{n}\left\{e_{t, j}-E\left(e_{t, j}\right)\right\} W_{h}(j) \\
= & \frac{1}{\sqrt{n}} \sum_{t=2}^{K+1} \sum_{j=1}^{t-1}\left\{e_{t, j}-E\left(e_{t, j}\right)\right\} W_{h}(j) \\
& +\frac{1}{\sqrt{n}} \sum_{t=K+2}^{n} \sum_{j=1}^{K}\left\{e_{t, j}-E\left(e_{t, j}\right)\right\} W_{h}(j),
\end{aligned}
$$

where the first summand above is $o_{p}(1)$ since $K$ is finite. Rewrite

$$
\begin{aligned}
Y_{t}:=\sum_{j=1}^{K} e_{t, j} W_{h}(j) & =1_{K+1}^{\prime} \times\left(\check{\varepsilon}_{t} \breve{\varepsilon}_{t-1} W_{h}(1), \cdots, \check{\varepsilon}_{t} \breve{\varepsilon}_{t-K} W_{h}(K), \kappa \check{\varepsilon}_{t} \frac{\partial \breve{\varepsilon}_{t}}{\partial \theta^{\prime}}\right)^{\prime} \\
& =: 1_{K+1}^{\prime} \times v_{t},
\end{aligned}
$$

where $1_{K+1}=(1, \cdots, 1)^{\prime} \in \mathcal{R}^{(K+1) \times 1}$ and $\kappa=-2 \sum_{j=1}^{K} E\left[\partial\left(\breve{\varepsilon}_{t} \breve{\varepsilon}_{t-j}\right) / \partial \theta^{\prime}\right] W_{h}(j)$. By the finiteness of $W_{h}(j)$ and $\kappa$ and the same argument as in Francq, Roy, and Zakoïan (2005, page 543), we have

$$
\frac{1}{\sqrt{n}} \sum_{t=K+2}^{n}\left(v_{t}-E v_{t}\right) \rightarrow_{d} N\left(0, \Omega^{*}\right) \text { as } n \rightarrow \infty, \text { where } \Omega^{*}=\lim _{n \rightarrow \infty} \operatorname{var}\left[\frac{1}{\sqrt{n}} \sum_{t=K+2}^{n} v_{t}\right]<\infty \text {. }
$$


Hence, it follows that for the second summand, $n^{-1 / 2} \sum_{t=K+2}^{n}\left(Y_{t}-E Y_{t}\right) \rightarrow{ }_{d} N(0, \breve{I})$ as $n \rightarrow \infty$, where

$$
\begin{aligned}
\breve{I} & =\lim _{n \rightarrow \infty} \operatorname{var}\left(\frac{1}{\sqrt{n}} \sum_{t=K+2}^{n} Y_{t}\right) \\
& =\lim _{n \rightarrow \infty} \frac{1}{n} \sum_{j=1}^{K} \sum_{k=1}^{K}\left(\sum_{t=K+2}^{n} \sum_{t^{\prime}=K+2}^{n} \operatorname{cov}\left(e_{t, j}, e_{t^{\prime}, k}\right)\right) W_{h}(j) W_{h}(k) \\
& =\lim _{n \rightarrow \infty} \frac{1}{n} \sum_{j=1}^{K} \sum_{k=1}^{K}\left(\sum_{d=K+2-n}^{n-K-2} \sum_{t=K+2+\max (0, d)}^{n+\min (0, d)} \operatorname{cov}\left(e_{t, j}, e_{t-d, k}\right)\right) W_{h}(j) W_{h}(k) \\
& =\lim _{n \rightarrow \infty} \sum_{j=1}^{K} \sum_{k=1}^{K}\left(\sum_{d=K+2-n}^{n-K-2} \frac{n-K-2-|d|}{n} \operatorname{cov}\left(e_{t, j}, e_{t-d, k}\right)\right) W_{h}(j) W_{h}(k) \\
& =\sigma_{h, K}^{2} .
\end{aligned}
$$

Thus, it follows that claim (a) holds.

PROOF OF CLAIM (b). First, as for (A9), we have

$$
\begin{aligned}
\bar{S}_{n}^{K}-E\left(\bar{S}_{n}^{K}\right) & =\frac{1}{\sqrt{n}} \sum_{j=1}^{K} \sum_{t=j+1}^{n}\left\{e_{t, j}-E\left(e_{t, j}\right)\right\} \psi_{j}(\lambda) \\
& =\frac{1}{\sqrt{n}} \sum_{t=2}^{K+1} \sum_{j=1}^{t-1}\left\{e_{t, j}-E\left(e_{t, j}\right)\right\} \psi_{j}(\lambda)+\frac{1}{\sqrt{n}} \sum_{t=K+2}^{n} G_{t}^{K},
\end{aligned}
$$

where the first term in (A12) is tight since each summand is tight, and

$$
G_{t}^{K}=\sum_{j=1}^{K}\left\{e_{t, j}-E\left(e_{t, j}\right)\right\} \psi_{j}(\lambda) \text {. }
$$

Next, we use Theorem 2.1 in Politis and Romano (1994) to prove the tightness of the second term in (A12). Note that $G_{t}^{K}$ is independent of $n$. We only need to verify that

(i) $E\left\|G_{t}^{K}\right\|^{2}<\infty$;

(ii) $\lim _{n \rightarrow \infty} \sum_{t=K+2}^{n} E\left[\left\langle G_{K+2}^{K}, G_{t}^{K}\right\rangle\right]=\sum_{t=K+2}^{\infty} E\left[\left\langle G_{K+2}^{K}, G_{t}^{K}\right\rangle\right]<\infty$, and the last series converges absolutely;

(iii) $\lim _{n \rightarrow \infty} \operatorname{var}\left[\left\langle\bar{S}_{n}^{K}-E\left(\bar{S}_{n}^{K}\right), h\right\rangle\right]=\sigma_{h, K}^{2}$.

The proof of (i) is trivial, and the proof of (iii) is directly from the one as for (A11). We now consider the proof of (ii). Note that

$$
\sum_{t=K+2}^{\infty}\left|E\left[\left\langle G_{K+2}^{K}, G_{t}^{K}\right\rangle\right]\right|=\sum_{t=K+2}^{\infty}\left|\sum_{j=1}^{K} \operatorname{cov}\left(e_{t, j}, e_{K+2, j}\right) P_{j}\right| .
$$

Using the same argument as for Lemma 3 in Francq and Zakoïan (1998), it is not hard to show that for each $j \in\{1, \cdots, K\}$, there exists a $\rho \in(0,1)$ such that

$$
\left|\operatorname{cov}\left(e_{t, j}, e_{K+2, j}\right)\right| \leq C\left\{\rho^{|t-K-2| / 2}+\left[\alpha_{y}\left(\left\lfloor\frac{|t-K-2|}{2}\right\rfloor\right)\right]^{\nu /(2+\nu)}\right\} .
$$


By (A13)-(A14), it follows that

$$
\sum_{t=K+2}^{\infty}\left|E\left[\left\langle G_{K+2}^{K}, G_{t}^{K}\right\rangle\right]\right| \leq C\left(\sum_{j=1}^{K} P_{j}\right) \sum_{s=0}^{\infty}\left\{\rho^{|s| / 2}+\left[\alpha_{y}\left(\left\lfloor\frac{|s|}{2}\right\rfloor\right)\right]^{\nu /(2+\nu)}\right\}<\infty
$$

which implies that (ii) holds. This completes the proof of claim (b).

Q.E.D.

PROOF OF CLAIM (c). First, by direct calculation, we have

$$
E\left\|R_{n}^{K}(\lambda)-E\left\{R_{n}^{K}(\lambda)\right\}\right\|^{2}=\frac{1}{n} \sum_{j=K+1}^{n-1} \sum_{t, t^{\prime}=j+1}^{n} \operatorname{cov}\left(e_{t, j}, e_{t^{\prime}, j}\right) P_{j} .
$$

Since $e_{t, j}=\check{\varepsilon}_{t} \check{\varepsilon}_{t-j}+z_{t j}$, there are four terms in $\operatorname{cov}\left(e_{t, j}, e_{t^{\prime}, j}\right)$. For simplicity, we only prove the conclusion for the term $\operatorname{cov}\left(z_{t j}, z_{t^{\prime} j}\right)$, since the proofs for other terms are similar. Note that for any $m \in\{1, \cdots, p+q\}$, the $m$-th entry of $z_{t j}$ satisfies that

$$
z_{t j, m}=O(1) \check{\varepsilon}_{t} \frac{\partial \varepsilon_{t}\left(\check{\theta}_{0}\right)}{\partial \theta_{m}}=O(1)\left[\sum_{i=0}^{\infty} c_{i} y_{t-i}\right]\left[\sum_{k=0}^{\infty} c_{k, m} y_{t-k}\right],
$$

where $c_{i}=O\left(\rho^{i}\right)$ and $c_{i, m}=O\left(\rho^{i}\right)$ for some $\rho \in(0,1)$. Then, for any $\left(m, m^{\prime}\right) \in\{1, \cdots, p+q\}^{2}$, we have

$$
\begin{aligned}
& \frac{1}{n} \sum_{j=K+1}^{n-1} \sum_{t, t^{\prime}=j+1}^{n} \operatorname{cov}\left(z_{t j, m}, z_{t^{\prime} j, m^{\prime}}\right) \\
& \leq O\left(\frac{1}{n}\right) \sum_{j=K+1}^{n-1} \sum_{t, t^{\prime}=j+1}^{n} \sum_{i, k, i^{\prime}, k^{\prime} \geq 0}\left|c_{i} c_{k, m^{\prime}} c_{i^{\prime}} c_{k^{\prime}, m^{\prime}}\right|\left|\operatorname{cov}\left(y_{t-i} y_{t-k}, y_{t^{\prime}-i^{\prime}} y_{t^{\prime}-k^{\prime}}\right)\right| P_{j} \\
& \leq O(1) \sum_{i, k, i^{\prime}, k^{\prime} \geq 0}\left|c_{i} c_{k, m^{\prime}} c_{i^{\prime}} c_{k^{\prime}, m^{\prime}}\right| \sum_{j=K+1}^{n-1}\left\{\frac{1}{n} \sum_{t, t^{\prime}=j+1}^{n}\left|\operatorname{cov}\left(y_{0} y_{i-k}, y_{t^{\prime}-t+i-i^{\prime}} y_{t^{\prime}-t+i-k^{\prime}}\right)\right|\right\} P_{j} .
\end{aligned}
$$

Furthermore, by Assumption 2, we can show that for any $i, k, i^{\prime}, k^{\prime}, j$,

$$
\begin{aligned}
& \frac{1}{n} \sum_{t, t^{\prime}=j+1}^{n}\left|\operatorname{cov}\left(y_{0} y_{i-k}, y_{t^{\prime}-t+i-i^{\prime}} y_{t^{\prime}-t+i-k^{\prime}}\right)\right| \\
& \leq \frac{1}{n} \sum_{t, t^{\prime}=j+1}^{n}\left\{\left|\operatorname{cum}\left(y_{0}, y_{i-k}, y_{t^{\prime}-t+i-i^{\prime}}, y_{t^{\prime}-t+i-k^{\prime}}\right)\right|\right. \\
& \left.\quad+\left|\gamma\left(t^{\prime}-t+i-i^{\prime}\right) \gamma\left(t^{\prime}-t+k-k^{\prime}\right)\right|+\left|\gamma\left(t^{\prime}-t+i-k^{\prime}\right) \gamma\left(t^{\prime}-t+k-i^{\prime}\right)\right|\right\} \\
& \leq \sum_{d=-(n-1-j)}^{n-1-j} \frac{n-1-j-|d|}{n}\left\{\left|\operatorname{cum}\left(y_{0}, y_{i-k}, y_{d+i-i^{\prime}}, y_{d+i-k^{\prime}}\right)\right|\right. \\
& \left.\quad+\left|\gamma\left(d+i-i^{\prime}\right) \gamma\left(d+k-k^{\prime}\right)\right|+\left|\gamma\left(d+i-k^{\prime}\right) \gamma\left(d+k-i^{\prime}\right)\right|\right\} \\
& \leq \sum_{s_{1}, s_{2}, s_{3}=-\infty}^{\infty}\left|\operatorname{cum}\left(y_{0}, y_{s_{1}}, y_{s_{2}}, y_{s_{3}}\right)\right|+2 \sum_{s=-\infty}^{\infty}[\gamma(s)]^{2}<\infty .
\end{aligned}
$$

Thus, it follows that

$$
\frac{1}{n} \sum_{j=K+1}^{n-1} \sum_{t, t^{\prime}=j+1}^{n} \operatorname{cov}\left(z_{t j, m}, z_{t^{\prime} j, m^{\prime}}\right) \leq O(1) \sum_{j=K+1}^{\infty} P_{j} \rightarrow 0 \text { as } K \rightarrow \infty .
$$


By (A15) and (A17), we know that $\lim _{K \rightarrow \infty} \lim _{n \rightarrow \infty} E\left\|R_{n}^{K}(\lambda)-E\left\{R_{n}^{K}(\lambda)\right\}\right\|^{2}=0$. Now, claim (c) $\quad 555$ follows directly from Chebyshev's inequality.

Proof OF COROLlary 1. Under $H_{0}$, we have $\theta_{0}=\breve{\theta}_{0}$ and $\gamma(j)=0$ for $j \geq 1$. Then, it is straightforward to see that $\left\|E\left\{\breve{S}_{n}(\lambda)\right\}\right\|=o(1)$. Thus, (i) follows directly from continuous mapping theorem. For (ii), since $n^{-1 / 2} \widetilde{S}_{n}(\lambda)-E\left\{n^{-1 / 2} \breve{S}_{n}(\lambda)\right\} \Rightarrow 0$ in $L_{2}[0, \pi]$ by Theorem 1 , it follows that

$$
\begin{aligned}
\frac{\widetilde{\mathrm{CM}}_{n}}{n} & =\left\|E\left[\frac{\breve{S}_{n}(\lambda)}{\sqrt{n}}\right]\right\|+\left\|\frac{\widetilde{S}_{n}(\lambda)}{\sqrt{n}}\right\|-\left\|E\left[\frac{\breve{S}_{n}(\lambda)}{\sqrt{n}}\right]\right\| \\
& =\sum_{j=1}^{\infty}\left[E\left(\check{\varepsilon}_{t} \breve{\varepsilon}_{t-j}\right)\right]^{2} P_{j}+o(1),
\end{aligned}
$$

which entails that (ii) holds.

Q.E.D.

Proof OF COROLlaRY 2. Rewrite

$$
\begin{aligned}
\widetilde{S}_{n}(\lambda) & =\bar{S}_{n}(\lambda)+\left[\widetilde{S}_{n}(\lambda)-\bar{S}_{n}(\lambda)\right] \\
& =\left[\bar{S}_{n}(\lambda)-E\left\{\bar{S}_{n}(\lambda)\right\}\right]+E\left\{\bar{S}_{n}(\lambda)\right\}+\left[\widetilde{S}_{n}(\lambda)-\bar{S}_{n}(\lambda)\right] .
\end{aligned}
$$

On one hand, by Assumptions 1-3, from the proof of Theorem 1, we have

$$
\bar{S}_{n}(\lambda)-E\left\{\bar{S}_{n}(\lambda)\right\} \Rightarrow S(\lambda) \text { and } E\left\|\widetilde{S}_{n}(\lambda)-\bar{S}_{n}(\lambda)\right\|^{2} \rightarrow 0 \text { as } n \rightarrow \infty .
$$

On the other hand, since $\check{\theta}_{0}=\theta_{0}$ by Assumption 4, we can show that under $H_{1 n}$,

$$
\begin{aligned}
E\left\{\bar{S}_{n}(\lambda)\right\} & =E\left\{\breve{S}_{n}(\lambda)\right\} \\
& =E\left[\sum_{j=1}^{n-1} \sqrt{n} \widehat{\gamma}(j) \psi_{j}(\lambda)\right] \\
& =\sum_{j=1}^{n-1} \sqrt{n} \gamma_{n}(j) \psi_{j}(\lambda) \\
& =\sum_{j=1}^{n-1} \frac{\gamma(0)}{2 \pi} \int_{-\pi}^{\pi}\left[g(\omega) e^{i j \omega} d \omega\right] \psi_{j}(\lambda) \rightarrow \frac{\gamma(0)}{2 \pi} \int_{0}^{\lambda} g(\omega) d \omega
\end{aligned}
$$

as $n \rightarrow \infty$, where $\gamma_{n}(j)$ is defined as in (5). Now, the conclusion holds from (A18)-(A20) and continuous mapping theorem.

Q.E.D.

Next, in order to prove Theorem 2, we need three more lemmas:

Lemma A6. Assume that Assumptions 1-3 hold and $b_{n}^{-1}=o(1)$. Then,

(i) $\left\|\theta_{n}^{*}-\breve{\theta}_{0}\right\|=o_{p}^{*}(1)$

(ii) $\sqrt{n}\left(\theta_{n}^{*}-\breve{\theta}_{0}\right)=O_{p}^{*}(1)$ with $\sqrt{n}\left(\theta_{n}^{*}-\breve{\theta}_{0}\right)=-\Sigma^{-1}\left[n^{-1 / 2} \sum_{t=1}^{n} w_{t}^{*} \frac{\partial l_{t}\left(\check{\theta}_{0}\right)}{\partial \theta}\right]+o_{p}^{*}(1)$. 
Proof. By the definition of $\theta_{n}^{*}$, we have

$$
\begin{aligned}
\theta_{n}^{*}-\breve{\theta}_{0} & =-\left[\frac{1}{n} \sum_{t=1}^{n} \frac{\partial^{2} \tilde{l}_{t}^{*}\left(\zeta_{n}\right)}{\partial \theta \partial \theta^{\prime}}\right]^{-1}\left[\frac{1}{n} \sum_{t=1}^{n} \frac{\partial \tilde{l}_{t}^{*}\left(\check{\theta}_{0}\right)}{\partial \theta}\right] \\
& =-\left[\frac{1}{n} \sum_{t=1}^{n} w_{t}^{*} \frac{\partial^{2} \tilde{l}_{t}\left(\zeta_{n}\right)}{\partial \theta \partial \theta^{\prime}}\right]^{-1}\left[\frac{1}{n} \sum_{t=1}^{n}\left(w_{t}^{*}-1\right) \frac{\partial \tilde{l}_{t}\left(\check{\theta}_{0}\right)}{\partial \theta}+\frac{1}{n} \sum_{t=1}^{n} \frac{\partial \tilde{l}_{t}\left(\check{\theta}_{0}\right)}{\partial \theta}\right] \\
& =:-\left[s_{1 n}\right]^{-1}\left[s_{2 n}+s_{3 n}\right]
\end{aligned}
$$

where $\zeta_{n}$ lies between $\theta_{n}^{*}$ and $\breve{\theta}_{0}$. First, by Lemma A1, it is straightforward to see that

$$
E^{*}\left\|s_{1 n}\right\| \leq \frac{1}{n} \sum_{t=1}^{n} E^{*}\left(w_{t}^{*}\right) \sup _{\Theta}\left\|\frac{\partial^{2} \widetilde{l}_{t}(\theta)}{\partial \theta \partial \theta^{\prime}}\right\|=\frac{1}{n} \sum_{t=1}^{n} \sup _{\Theta}\left\|\frac{\partial^{2} l_{t}(\theta)}{\partial \theta \partial \theta^{\prime}}\right\|+o_{p}(1)=O_{p}(1),
$$

which entails $s_{1 n}=O_{p}^{*}(1)$. Next, by direct calculation and Lemma A1, we have

$$
\begin{aligned}
E^{*}\left[s_{2 n} s_{2 n}^{\prime}\right] & =\frac{1}{n^{2}} \sum_{s=1}^{L_{n}}\left[\sum_{t, t^{\prime} \in B_{s}} \frac{\partial \widetilde{l}_{t}\left(\check{\theta}_{0}\right)}{\partial \theta} \frac{\partial \widetilde{\partial}_{t^{\prime}}\left(\check{\theta}_{0}\right)}{\partial \theta^{\prime}}\right] \\
& =\frac{1}{n^{2}} \sum_{s=1}^{L_{n}}\left[\sum_{t, t^{\prime} \in B_{s}} \frac{\partial l_{t}\left(\check{\theta}_{0}\right)}{\partial \theta} \frac{\partial l_{t^{\prime}}\left(\check{\theta}_{0}\right)}{\partial \theta^{\prime}}\right]+o_{p}(1)=: s_{4 n}+o_{p}(1),
\end{aligned}
$$

where $B_{s}$ is defined in step 1 of the BRW procedure in Section 3. Moreover, since $l_{t}(\theta)$ is stationary and $E \partial l_{t}\left(\breve{\theta}_{0}\right) / \partial \theta=0$ by Lemma A3(i), it is straightforward to see that

$$
E\left(s_{4 n}\right)=\frac{b_{n}}{n^{2}} \sum_{s=1}^{L_{n}} \operatorname{var}\left[\frac{1}{\sqrt{b_{n}}} \sum_{t \in B_{s}} \frac{\partial l_{t}\left(\check{\theta}_{0}\right)}{\partial \theta}\right]=\frac{b_{n} L_{n}}{n^{2}} \operatorname{var}\left[\frac{1}{\sqrt{b_{n}}} \sum_{t=1}^{b_{n}} \frac{\partial l_{t}\left(\check{\theta}_{0}\right)}{\partial \theta}\right]=O\left(\frac{1}{n}\right),
$$

where the last equality holds due to the fact that $b_{n}^{-1}=o(1)$ and (9). Then, by (A21)-(A22), we have $s_{2 n}=O_{p}^{*}\left(n^{-1 / 2}\right)$. Note that $s_{3 n}=o_{p}(1)$ by the ergodic theorem and Lemmas A1 and A3(i). Thus, it follows that (i) holds. Consequently, by Lemma A1, it is not hard to show that

$$
\frac{1}{n} \sum_{t=1}^{n}\left(w_{t}^{*}-1\right) \frac{\partial^{2} \widetilde{l}_{t}\left(\zeta_{n}\right)}{\partial \theta \partial \theta^{\prime}}=o_{p}^{*}(1) \text { and } \frac{1}{n} \sum_{t=1}^{n} \frac{\partial^{2} \widetilde{l}_{t}\left(\zeta_{n}\right)}{\partial \theta \partial \theta^{\prime}}=\Sigma+o_{p}^{*}(1)
$$

and hence $s_{1 n}=\Sigma+o_{p}^{*}(1)$. Note that $\sqrt{n} s_{2 n}=O_{p}^{*}(1)$ and $\sqrt{n} s_{3 n}=O_{p}(1)$ by Lemma A3(ii). Thus, it follows that (ii) holds.

LEMma A7. Assume that Assumptions 1-3 hold, $b_{n}^{-1}=o(1)$, and $b_{n} n^{-1}=o(1)$. Then, $E^{*} \| \widetilde{Z}_{n}(\gamma)-$ $\bar{Z}_{n}(\gamma) \|^{2}=o_{p}(1)$, where $\widetilde{Z}_{n}(\gamma)$ is defined in (6), and

$$
\bar{Z}_{n}(\gamma)=\sum_{j=1}^{n-1}\left[\frac{1}{\sqrt{n}} \sum_{t=1+j}^{n}\left(w_{t}^{*}-1\right) E\left(e_{t, j}\right)\right] \psi_{j}(\lambda)
$$

Proof. Note that

$$
E^{*}\left\|\widetilde{Z}_{n}(\gamma)-\bar{Z}_{n}(\gamma)\right\|^{2} \leq 2 E^{*}\left\|\widetilde{Z}_{n}(\gamma)-\breve{Z}_{n}(\gamma)\right\|^{2}+2 E^{*}\left\|\breve{Z}_{n}(\gamma)-\bar{Z}_{n}(\gamma)\right\|^{2}
$$

where

$$
\check{Z}_{n}(\gamma)=\sum_{j=1}^{n-1}\left[\frac{1}{\sqrt{n}} \sum_{t=1+j}^{n} \frac{\left(w_{t}^{*}-1\right)(n-j)}{n} E\left(\check{\varepsilon}_{t} \check{\varepsilon}_{t-j}\right)\right] \psi_{j}(\lambda)
$$


By direct calculation, we have

$$
\begin{aligned}
E^{*}\left\|\widetilde{Z}_{n}(\gamma)-\check{Z}_{n}(\gamma)\right\|^{2} & =\sum_{j=1}^{n-1}\left\{\frac{1}{n} E^{*}\left[\sum_{t=1+j}^{n}\left(w_{t}^{*}-1\right) d_{n j}\right]^{2}\right\} P_{j} \\
& =\sum_{j=1}^{n-1}\left\{\frac{1}{n} \sum_{s=1}^{L_{n}}\left[\sum_{t \in B_{s} \cap[1+j, n]} d_{n j}\right]^{2}\right\} P_{j} \\
& \leq \sum_{j=1}^{n-1}\left\{\frac{L_{n} b_{n}^{2}}{n} d_{n j}^{2}\right\} P_{j} \\
& =\frac{b_{n}}{n} \sum_{j=1}^{n-1}\left(\sqrt{n} d_{n j}\right)^{2} P_{j}
\end{aligned}
$$

where $d_{n j}=n^{-1} \sum_{t^{\prime}=1+j}^{n}\left[\widetilde{\varepsilon}_{t^{\prime}} \widetilde{\varepsilon}_{t^{\prime}-j}-E\left(\breve{\varepsilon}_{t^{\prime}} \breve{\varepsilon}_{t^{\prime}-j}\right)\right]$. By Lemma A1, it is straightforward to see that

$$
\sqrt{n} d_{n j}=\frac{1}{\sqrt{n}} \sum_{t=1+j}^{n}\left[\breve{\varepsilon}_{t} \breve{\varepsilon}_{t-j}-E\left(\breve{\varepsilon}_{t} \breve{\varepsilon}_{t-j}\right)\right]+o_{p}(1)
$$

Next, by Taylor's expansion, we have

$$
\breve{\varepsilon}_{t} \breve{\varepsilon}_{t-j}=\breve{\varepsilon}_{t} \breve{\varepsilon}_{t-j}+\frac{\partial\left(\breve{\varepsilon}_{t} \breve{\varepsilon}_{t-j}\right)}{\partial \theta^{\prime}}\left(\theta_{n}-\breve{\theta}_{0}\right)+\left(\theta_{n}-\breve{\theta}_{0}\right)^{\prime}\left[\left.\frac{1}{2} \frac{\partial^{2}\left(\varepsilon_{t}(\theta) \varepsilon_{t-j}(\theta)\right)}{\partial \theta \partial \theta^{\prime}}\right|_{\theta=\zeta_{n}}\right]\left(\theta_{n}-\breve{\theta}_{0}\right),
$$

where $\zeta_{n}$ lies between $\theta_{n}$ and $\breve{\theta}_{0}$. Note that $\sqrt{n}\left(\theta_{n}-\breve{\theta}_{0}\right)=O_{p}(1)$ by Lemma A3(ii). Thus, by (A25) it follows that for all $j \in\{1, \cdots, n-1\}$,

$$
\begin{aligned}
\sqrt{n} d_{n j}= & \frac{1}{\sqrt{n}} \sum_{t=1+j}^{n}\left[\check{\varepsilon}_{t} \breve{\varepsilon}_{t-j}-E\left(\breve{\varepsilon}_{t} \breve{\varepsilon}_{t-j}\right)\right] \\
& +\frac{1}{n} \sum_{t=1+j}^{n} \frac{\partial\left(\breve{\varepsilon}_{t} \breve{\varepsilon}_{t-j}\right)}{\partial \theta^{\prime}}\left[\sqrt{n}\left(\theta_{n}-\breve{\theta}_{0}\right)\right]+o_{p}(1) \\
= & \frac{1}{\sqrt{n}} \sum_{t=1+j}^{n}\left[\check{\varepsilon}_{t} \check{\varepsilon}_{t-j}-E\left(\check{\varepsilon}_{t} \breve{\varepsilon}_{t-j}\right)\right]+O_{p}(1) .
\end{aligned}
$$

As for (A17), we can show that for all $j \in\{1, \cdots, n-1\}$,

$$
E\left\{\frac{1}{\sqrt{n}} \sum_{t=1+j}^{n}\left[\breve{\varepsilon}_{t} \breve{\varepsilon}_{t-j}-E\left(\breve{\varepsilon}_{t} \breve{\varepsilon}_{t-j}\right)\right]\right\}^{2}=\frac{1}{n} \sum_{t, t^{\prime}=1+j}^{n} \operatorname{cov}\left(\breve{\varepsilon}_{t} \breve{\varepsilon}_{t-j}, \breve{\varepsilon}_{t^{\prime}} \breve{\varepsilon}_{t^{\prime}-j}\right)=O(1) .
$$

Thus, by (A26), we know that $\sqrt{n} d_{n j}=O_{p}(1)$ for all $j$. Since $b_{n} n^{-1}=o(1)$ and $\sum_{j=1}^{\infty} P_{j}<\infty$, by (A24), it entails that

$$
E^{*}\left\|\widetilde{Z}_{n}(\gamma)-\breve{Z}_{n}(\gamma)\right\|^{2}=\frac{b_{n}}{n} \sum_{j=1}^{n-1} O_{p}\left(P_{j}\right)=o_{p}(1)
$$


Next, since $E\left(e_{t, j}\right)=E\left(\breve{\varepsilon}_{t} \breve{\varepsilon}_{t-j}\right)$ and $b_{n} n^{-1}=o(1)$, it is straightforward to see that

625

$$
\begin{aligned}
E^{*}\left\|\check{Z}_{n}(\gamma)-\bar{Z}_{n}(\gamma)\right\|^{2} & =E^{*}\left\|\sum_{j=1}^{n-1}\left[\frac{j}{n^{3 / 2}} \sum_{t=1+j}^{n}\left(w_{t}^{*}-1\right) E\left(\check{\varepsilon}_{t} \check{\varepsilon}_{t-j}\right)\right] \psi_{j}(\lambda)\right\|^{2} \\
& =\sum_{j=1}^{n-1} \frac{j^{2}}{n^{3}} E^{*}\left[\sum_{t=1+j}^{n}\left(w_{t}^{*}-1\right) E\left(\check{\varepsilon}_{t} \check{\varepsilon}_{t-j}\right)\right]^{2} P_{j} \\
& =\sum_{j=1}^{n-1} \frac{j^{2}}{n^{3}} \sum_{s=1}^{L_{n}}\left[\sum_{t \in B_{s} \cap[1+j, n]} E\left(\check{\varepsilon}_{t} \check{\varepsilon}_{t-j}\right)\right]^{2} P_{j} \\
& \leq \sum_{j=1}^{n-1} \frac{j^{2}}{n^{3}} L_{n} b_{n}^{2} P_{j} \\
& =O\left(b_{n} n^{-1}\right)=o(1) .
\end{aligned}
$$

Now, the conclusion follows directly from (A23) and (A27)-(A28).

Lemma A8. Suppose that Assumptions 1-3 hold, $b_{n}^{-1}=o(1)$, and $(\log n) b_{n} n^{-1}=o(1)$. Then,

$$
E^{*}\left\|\sum_{j=1}^{n-1}\left[\frac{1}{\sqrt{n}} \sum_{t=1}^{j}\left(w_{t}^{*}-1\right) \widetilde{z}_{t j}\right] \psi_{j}(\lambda)\right\|^{2}=o_{p}(1)
$$

where $\widetilde{z}_{t j}$ is defined in the same way as $z_{t j}$ in (3) with $\widetilde{l}_{t}\left(\check{\theta}_{0}\right)$ replacing $l_{t}\left(\check{\theta}_{0}\right)$.

Proof. By direct calculation, we have

$$
\begin{aligned}
E^{*}\left\|\sum_{j=1}^{n-1}\left[\frac{1}{\sqrt{n}} \sum_{t=1}^{j}\left(w_{t}^{*}-1\right) \widetilde{z}_{t j}\right] \psi_{j}(\lambda)\right\|^{2} & =\sum_{j=1}^{n-1} \frac{1}{n} E^{*}\left(\sum_{t=1}^{j}\left(w_{t}^{*}-1\right) \widetilde{z}_{t j}\right)^{2} P_{j} \\
& =\sum_{j=1}^{n-1} \frac{1}{n} \sum_{s=1}^{L_{n}}\left(\sum_{t \in B_{s} \cap[1, j]} \widetilde{z}_{t j}\right)^{2} P_{j} .
\end{aligned}
$$

By Lemma A1, it is straightforward to see that

$$
\sum_{j=1}^{n-1} \frac{1}{n} \sum_{s=1}^{L_{n}}\left(\sum_{t \in B_{s} \cap[1, j]} \widetilde{z}_{t j}\right)^{2} P_{j}=\sum_{j=1}^{n-1} \frac{1}{n} \sum_{s=1}^{L_{n}}\left(\sum_{t \in B_{s} \cap[1, j]} z_{t j}\right)^{2} P_{j}+o_{p}(1)=: H_{n}+o_{p}(1) .
$$

Note that $\sum_{j=1}^{\infty} P_{j}<\infty$. For $\forall \varepsilon>0$, there exists a $j_{0}(\varepsilon)>0$ such that

$$
\sum_{j=j_{0}+1}^{\infty} P_{j}<\varepsilon
$$


Since $b_{n} \rightarrow \infty$ as $n \rightarrow \infty$, we rewrite

$$
\begin{aligned}
H_{n}= & \sum_{j=1}^{j_{0}} \frac{1}{n} \sum_{s=1}^{L_{n}}\left(\sum_{t \in B_{s} \cap[1, j]} z_{t j}\right)^{2} P_{j}+\sum_{j=j_{0}+1}^{b_{n}} \frac{1}{n} \sum_{s=1}^{L_{n}}\left(\sum_{t \in B_{s} \cap[1, j]} z_{t j}\right)^{2} P_{j} \\
& +\sum_{j=b_{n}+1}^{n-1} \frac{1}{n} \sum_{s=1}^{L_{n}}\left(\sum_{t \in B_{s} \cap[1, j]} z_{t j}\right)^{2} P_{j} \\
= & : H_{1 n}+H_{2 n}+H_{3 n} .
\end{aligned}
$$

First, for $H_{1 n}$, we know that as $n$ is large enough,

$$
E H_{1 n} \leq \sum_{j=1}^{j_{0}} \frac{1}{n} \sum_{s=1}^{L_{n}} O\left(j_{0}^{2}\right) P_{j}=O\left(\frac{L_{n}}{n}\right)<\varepsilon .
$$

Next, for $H_{2 n}$, direct calculation gives us that

$$
H_{2 n}=\sum_{j=j_{0}+1}^{b_{n}} \frac{1}{n} \sum_{s=1}^{1}\left(\sum_{t \in B_{s} \cap[1, j]} z_{t j}\right)^{2} P_{j}=\sum_{j=j_{0}+1}^{b_{n}} \frac{1}{n}\left(\sum_{t \in B_{1}} z_{t j}\right)^{2} P_{j} .
$$

By Lemma 3 in Francq and Zakoïan (1998), it follows that as $n$ is large enough,

$$
E H_{2 n}=\sum_{j=j_{0}+1}^{b_{n}} \frac{b_{n}}{n} E\left(\frac{1}{\sqrt{b_{n}}} \sum_{t=1}^{b_{n}} z_{t j}\right)^{2} P_{j}=\sum_{j=j_{0}+1}^{b_{n}} \frac{b_{n}}{n} O\left(P_{j}\right) \leq O\left(\frac{b_{n}}{n} \varepsilon\right)<\varepsilon .
$$

Third, for $H_{3 n}$, we truncate it as

$$
\begin{aligned}
H_{3 n} & =\frac{1}{n} \sum_{s^{\prime}=2}^{L_{n}} \sum_{j \in B_{s^{\prime}}} \sum_{s=1}^{L_{n}}\left(\sum_{t \in B_{s} \cap[1, j]} z_{t j}\right)^{2} P_{j} \\
& =\frac{1}{n} \sum_{s^{\prime}=2}^{L_{n}} \sum_{j \in B_{s^{\prime}}}\left(\sum_{s<s^{\prime}}+\sum_{s=s^{\prime}}\right)\left(\sum_{t \in B_{s} \cap[1, j]} z_{t j}\right)^{2} P_{j},
\end{aligned}
$$

where the summand in the case of $s>s^{\prime}$ is zero since $B_{s} \cap[1, j]=\emptyset$. As for (A31), by the stationarity of $z_{t j}$, we can show that

$$
\begin{aligned}
E\left[\frac{1}{n} \sum_{s^{\prime}=2}^{L_{n}} \sum_{j \in B_{s^{\prime}}} \sum_{s<s^{\prime}}\left(\sum_{t \in B_{s} \cap[1, j]} z_{t j}\right)^{2} P_{j}\right] & =\frac{1}{n} \sum_{s^{\prime}=2}^{L_{n}} \sum_{j \in B_{s^{\prime}}} \sum_{s<s^{\prime}} E\left(\sum_{t \in B_{s}} z_{t j}\right)^{2} P_{j} \\
& =\frac{b_{n}}{n} \sum_{s^{\prime}=2}^{L_{n}} \sum_{j \in B_{s^{\prime}}} \sum_{s<s^{\prime}} O\left(P_{j}\right) \\
& \leq \frac{b_{n} L_{n}}{n} \sum_{s^{\prime}=2}^{L_{n}} \sum_{j \in B_{s^{\prime}}} O\left(P_{j}\right) \\
& \leq \sum_{j=j_{0}+1}^{\infty} O\left(P_{j}\right)<\varepsilon .
\end{aligned}
$$


Furthermore, since $(\log n) b_{n} n^{-1}=o(1)$, it is not hard to see that

$$
\begin{aligned}
E\left[\frac{1}{n} \sum_{s^{\prime}=2}^{L_{n}} \sum_{j \in B_{s^{\prime}}} \sum_{s=s^{\prime}}\left(\sum_{t \in B_{s} \cap[1, j]} z_{t j}\right)^{2} P_{j}\right] & =\frac{1}{n} \sum_{s^{\prime}=2}^{L_{n}} \sum_{j \in B_{s^{\prime}}} O\left(b_{n}^{2}\right) P_{j} \\
& =\frac{1}{n} \sum_{j=b_{n}+1}^{n-1} O\left(b_{n}^{2}\right) \frac{1}{j^{2}} \\
& \leq \frac{b_{n}}{n} \sum_{j=b_{n}+1}^{n-1} O(1) \frac{1}{j} \\
& =O\left(\frac{b_{n} \log n}{n}\right)<\varepsilon .
\end{aligned}
$$

Now, the conclusion follows from (A29)-(A34).

Proof of TheOREM 2. By Taylor's expansion we have

665

$$
\widetilde{\varepsilon}_{t}^{*} \widetilde{\varepsilon}_{t-j}^{*}=\widetilde{\varepsilon}_{t} \widetilde{\varepsilon}_{t-j}+\frac{\partial\left(\widetilde{\varepsilon}_{t} \widetilde{\varepsilon}_{t-j}\right)}{\partial \theta^{\prime}}\left(\theta_{n}^{*}-\theta_{n}\right)+\left(\theta_{n}^{*}-\theta_{n}\right)^{\prime}\left[\left.\frac{1}{2} \frac{\partial^{2}\left(\widetilde{\varepsilon}_{t}(\theta) \widetilde{\varepsilon}_{t-j}(\theta)\right)}{\partial \theta \partial \theta^{\prime}}\right|_{\theta=\zeta_{n}}\right]\left(\theta_{n}^{*}-\theta_{n}\right),
$$

where $\zeta_{n}$ lies between $\theta_{n}^{*}$ and $\theta_{n}$. Then, it follows that

$$
\begin{aligned}
\widetilde{S}_{n}^{*}(\lambda)-\widetilde{S}_{n}(\lambda)= & \sum_{j=1}^{n-1} \frac{1}{\sqrt{n}}\left[\sum_{t=1+j}^{n}\left(w_{t}^{*}-1\right) \widetilde{\varepsilon}_{t} \widetilde{\varepsilon}_{t-j}\right] \psi_{j}(\lambda)+I_{1 n}^{*}(\lambda)\left[\sqrt{n}\left(\theta_{n}^{*}-\theta_{n}\right)\right] \\
& +\left[\sqrt{n}\left(\theta_{n}^{*}-\theta_{n}\right)^{\prime}\right] I_{2 n}^{*}(\lambda)\left[\sqrt{n}\left(\theta_{n}^{*}-\theta_{n}\right)\right],
\end{aligned}
$$

where

$$
\begin{aligned}
& I_{1 n}^{*}(\lambda)=\sum_{j=1}^{n-1} \frac{1}{n} \sum_{t=1+j}^{n} w_{t}^{*} \frac{\partial\left(\widetilde{\varepsilon}_{t} \widetilde{\varepsilon}_{t-j}\right)}{\partial \theta^{\prime}} \psi_{j}(\lambda), \\
& I_{2 n}^{*}(\lambda)=\sum_{j=1}^{n-1} \frac{1}{n^{3 / 2}} \sum_{t=1+j}^{n} w_{t}^{*}\left[\left.\frac{1}{2} \frac{\partial^{2}\left(\widetilde{\varepsilon}_{t}(\theta) \widetilde{\varepsilon}_{t-j}(\theta)\right)}{\partial \theta \partial \theta^{\prime}}\right|_{\theta=\zeta_{n}}\right] \psi_{j}(\lambda) .
\end{aligned}
$$

By Lemma A4, we can easily show that

$$
E^{*}\left\|I_{1 n}^{*}(\lambda)-\sum_{j=1}^{n-1} E\left[\frac{\partial\left(\check{\varepsilon}_{t} \check{\varepsilon}_{t-j}\right)}{\partial \theta^{\prime}}\right] \psi_{j}(\lambda)\right\|^{2}=O_{p}\left(b_{n} n^{-1}\right) .
$$

On the other hand, it is straightforward to see that

$$
E^{*}\left\|I_{2 n}^{*}(\lambda)\right\|^{2}=O_{p}\left(n^{-1}\right) .
$$

Since $\sqrt{n}\left(\theta_{n}^{*}-\theta_{n}\right)=O_{p}^{*}(1)$ by Lemma A3(ii) and Lemma A6(ii), under (A35)-(A37), we have

$$
\begin{aligned}
\widetilde{S}_{n}^{*}(\lambda)-\widetilde{S}_{n}(\lambda)= & \sum_{j=1}^{n-1} \frac{1}{\sqrt{n}}\left[\sum_{t=1+j}^{n}\left(w_{t}^{*}-1\right) \widetilde{\varepsilon}_{t} \widetilde{\varepsilon}_{t-j}\right] \psi_{j}(\lambda) \\
& +\left\{\sum_{j=1}^{n-1} E\left[\frac{\partial\left(\widetilde{\varepsilon}_{t} \widetilde{\varepsilon}_{t-j}\right)}{\partial \theta^{\prime}}\right] \psi_{j}(\lambda)\right\}\left[\sqrt{n}\left(\theta_{n}^{*}-\theta_{n}\right)\right]+\text { negligible terms. }
\end{aligned}
$$


Moreover, by Lemma A3(ii), Lemma A6(ii) and (A3), we have

$$
\begin{aligned}
\sqrt{n}\left(\theta_{n}^{*}-\theta_{n}\right) & =-\Sigma^{-1}\left[\frac{1}{\sqrt{n}} \sum_{t=1}^{n}\left(w_{t}^{*}-1\right) \frac{\partial l_{t}\left(\check{\theta}_{0}\right)}{\partial \theta}\right]+o_{p}^{*}(1) \\
& =-\Sigma^{-1}\left[\frac{1}{\sqrt{n}} \sum_{t=1}^{n}\left(w_{t}^{*}-1\right) \frac{\partial \widetilde{l}_{t}\left(\check{\theta}_{0}\right)}{\partial \theta}\right]+o_{p}^{*}(1) .
\end{aligned}
$$

By (A38)-(A39) and Lemma A8, it follows that

$$
\widetilde{S}_{n}^{*}(\lambda)-\widetilde{S}_{n}(\lambda)=\sum_{j=1}^{n-1} \frac{1}{\sqrt{n}}\left[\sum_{t=1+j}^{n}\left(w_{t}^{*}-1\right) \widetilde{e}_{t, j}\right] \psi_{j}(\lambda)+\text { negligible terms }
$$

where $\widetilde{e}_{t, j}=\widetilde{\varepsilon}_{t} \widetilde{\varepsilon}_{t-j}+\widetilde{z}_{t j}$ and $\widetilde{z}_{t j}$ is defined as in Lemma A8.

Let $\breve{\gamma}^{*}(j)=n^{-1}\left\{\sum_{t=1+j}^{n}\left(w_{t}^{*}-1\right)\left[\widetilde{e}_{t, j}-E\left(e_{t, j}\right)\right]\right\}$. By (A40) and Lemma A7, it follows that

$$
\Delta_{n}(\lambda)=\sqrt{n} \sum_{j=1}^{n-1} \breve{\gamma}^{*}(j) \psi_{j}(\lambda)+\text { negligible terms }=: \breve{S}_{n}^{*}(\lambda)+\text { negligible terms }
$$

Finally, for each fixed integer $K \in\{1, \cdots, n-1\}$, we rewrite

$$
\breve{S}_{n}^{*}(\lambda)=\sqrt{n} \sum_{j=1}^{K} \breve{\gamma}^{*}(j) \psi_{j}(\lambda)+\sqrt{n} \sum_{j=K+1}^{n-1} \breve{\gamma}^{*}(j) \psi_{j}(\lambda)=: \breve{S}_{n}^{K *}(\lambda)+\breve{R}_{n}^{K *}(\lambda)
$$

Then, as in Shao (2011a), the conclusion holds from the following three claims:

(d). For any $h \in L_{2}[0, \pi]$, the finite dimensional distributions of $\left\langle\breve{S}_{n}^{K *}, h\right\rangle$ converge to those of $\left\langle S^{K}(\lambda), h\right\rangle$ in probability conditional on $\chi_{n}$.

(e). For $\forall \varepsilon>0, \lim _{K \rightarrow \infty} \lim _{n \rightarrow \infty} P^{*}\left(\left\|\breve{R}_{n}^{K *}(\lambda)\right\|>\varepsilon\right)=0$ in probability conditional on $\chi_{n}$.

(f). The sequence $\left\{\breve{S}_{n}^{*}(\lambda)\right\}$ is tight in probability conditional on $\chi_{n}$.

The proofs of claims (e) and (f) are similar to these of part (a,ii) and part (b) in Shao (2011a, p.222). Thus, we only need to prove claim (d).

Q.E.D.

ProOF OF CLAIM (d). Let $G_{t}^{K *}=\sum_{j=1}^{K}\left(w_{t}^{*}-1\right)\left[\widetilde{e}_{t, j}-E\left(e_{t, j}\right)\right] \psi_{j}(\lambda)$. As for (A9), it suffices to ${ }_{695}$ show the asymptotic normality of $J_{n}^{K *}$, where

$$
\begin{aligned}
J_{n}^{K *}=\sum_{t=K+2}^{n} \frac{1}{\sqrt{n}}\left\langle G_{t}^{K *}, h\right\rangle & =\sum_{t=K+2}^{n} \frac{1}{\sqrt{n}} \sum_{j=1}^{K}\left(w_{t}^{*}-1\right)\left[\widetilde{e}_{t, j}-E\left(e_{t, j}\right)\right] W_{h}(j) \\
& =\sum_{s=1}^{L_{n}} \frac{\delta_{s}-1}{\sqrt{n}} \sum_{t \in B_{s} \cap[K+2, n]} \sum_{j=1}^{K}\left[\widetilde{e}_{t, j}-E\left(e_{t, j}\right)\right] W_{h}(j) \\
& =: \sum_{s=1}^{L_{n}} H_{s n}^{*} .
\end{aligned}
$$

Note that conditional on $\chi_{n},\left\{H_{s n}^{*}\right\}$ is a sequence of independent random variables. Thus, we only need 700 to verify that

(i) $\lim _{n \rightarrow \infty} \operatorname{var}^{*}\left(J_{n}^{K *}\right) \rightarrow_{p} \sigma_{h, K}^{2}$

(ii) $\lim _{n \rightarrow \infty} \sum_{s=1}^{L_{n}} E^{*}\left\{\left|H_{s n}^{*}\right|^{2} I\left(\left|H_{s n}^{*}\right|>\varepsilon\right)\right\} \rightarrow{ }_{p} 0$. 
Without loss of generality, we assume that $K+2 \leq b_{n}$. For (i), by Lemma A1, Taylor's expansion, and

Lemma A3(ii), it is not hard to show that

$$
\begin{aligned}
\operatorname{var}^{*}\left(J_{n}^{K *}\right) & =\frac{1}{n} \sum_{s=1}^{L_{n}}\left\{\sum_{t \in B_{s} \cap[K+2, n]} \sum_{j=1}^{K}\left[\widetilde{e}_{t, j}-E\left(e_{t, j}\right)\right] W_{h}(j)\right\}^{2} \\
& =\frac{1}{L_{n}} \sum_{s=2}^{L_{n}}\left\{\frac{1}{\sqrt{b_{n}}} \sum_{t \in B_{s}} \sum_{j=1}^{K}\left[\breve{e}_{t, j}-E\left(e_{t, j}\right)\right] W_{h}(j)\right\}^{2}+o_{p}(1) \\
& =\frac{1}{L_{n}} \sum_{s=2}^{L_{n}}\left\{\frac{1}{\sqrt{b_{n}}} \sum_{t \in B_{s}} \sum_{j=1}^{K}\left[e_{t, j}-E\left(e_{t, j}\right)\right] W_{h}(j)\right\}^{2}+O_{p}\left(\frac{b_{n}}{n}\right)+o_{p}(1) \\
& =: Z_{n}+o_{p}(1),
\end{aligned}
$$

where $\breve{e}_{t, j}=\breve{\varepsilon}_{t} \breve{\varepsilon}_{t-j}+z_{t j}$. As for (A11), we have $E Z_{n} \rightarrow \sigma_{h, k}^{2}$ as $n \rightarrow \infty$. Thus, we only need to prove that $\operatorname{var}\left(Z_{n}\right) \rightarrow 0$ as $n \rightarrow \infty$. By direct calculation, we have

$$
\begin{aligned}
\operatorname{var}\left(Z_{n}\right)= & \frac{1}{n^{2}} \sum_{s, s^{\prime}=1}^{L_{n}} \sum_{t_{1}, t_{2} \in B_{s}} \sum_{t_{1}^{\prime}, t_{2}^{\prime} \in B_{s^{\prime}}} \sum_{j_{1}, j_{2}=1}^{K} \sum_{j_{1}^{\prime}, j_{2}^{\prime}=1}^{K} C\left(t_{1}, t_{2}, t_{1}^{\prime}, t_{2}^{\prime}, j_{1}, j_{2}, j_{1}^{\prime}, j_{2}^{\prime}\right) \\
& \times W_{h}\left(j_{1}\right) W_{h}\left(j_{2}\right) W_{h}\left(j_{1}^{\prime}\right) W_{h}\left(j_{2}^{\prime}\right) \\
= & \frac{1}{n^{2}} \sum_{s, s^{\prime}=1}^{L_{n}} z\left(s, s^{\prime}\right)
\end{aligned}
$$

where $\gamma_{e}(j)=E\left(e_{t, j}\right)$ and $C\left(t_{1}, t_{2}, t_{1}^{\prime}, t_{2}^{\prime}, j_{1}, j_{2}, j_{1}^{\prime}, j_{2}^{\prime}\right)$ equals to

$$
\operatorname{cov}\left\{\left[\left(e_{t_{1}, j_{1}}-\gamma_{e}\left(j_{1}\right)\right)\left(e_{t_{2}, j_{2}}-\gamma_{e}\left(j_{2}\right)\right)\right],\left[\left(e_{t_{1}^{\prime}, j_{1}^{\prime}}-\gamma_{e}\left(j_{1}^{\prime}\right)\right)\left(e_{t_{2}^{\prime}, j_{2}^{\prime}}-\gamma_{e}\left(j_{2}^{\prime}\right)\right)\right]\right\} .
$$

$$
\operatorname{var}\left(Z_{n}\right)=\frac{1}{n^{2}} \sum_{s=1}^{L_{n}} \sum_{\left|s^{\prime}-s\right| \leq 1} z\left(s, s^{\prime}\right)+\frac{1}{n^{2}} \sum_{s=1}^{L_{n}} \sum_{\left|s^{\prime}-s\right|>1} z\left(s, s^{\prime}\right) .
$$

Fort the first summand in (A41), since $b_{n}=o\left(n^{1 / 3}\right)$, it is straightforward to see that

$$
\frac{1}{n^{2}} \sum_{s=1}^{L_{n}} \sum_{\left|s^{\prime}-s\right| \leq 1} z\left(s, s^{\prime}\right)=O\left(\frac{L_{n} b_{n}^{4}}{n^{2}}\right)=O\left(\frac{b_{n}^{3}}{n}\right)=o(1) .
$$

Next, for the second summand in (A41), $C\left(t_{1}, t_{2}, t_{1}^{\prime}, t_{2}^{\prime}, j_{1}, j_{2}, j_{1}^{\prime}, j_{2}^{\prime}\right)$ can be divided into 16 terms, since 
proofs for other terms are similar. In view of (A16), for any $\left(m_{1}, m_{2}, m_{1}^{\prime}, m_{2}^{\prime}\right) \in\{1, p+q\}^{4}$, we have

$$
\begin{aligned}
& \left|\operatorname{cov}\left[z_{t_{1} j_{1}, m_{1}} z_{t_{2} j_{2}, m_{2}}, z_{t_{1}^{\prime} j_{1}^{\prime}, m_{1}^{\prime}} z_{t_{2}^{\prime} j_{2}^{\prime}, m_{2}^{\prime}}\right]\right| \\
= & \left|\sum_{i_{1}, k_{1}, i_{2}, k_{2}, i_{1}^{\prime}, k_{1}^{\prime}, i_{2}^{\prime}, k_{2}^{\prime}} c_{i_{1}} c_{k_{1}, m_{1}} c_{i_{2}} c_{k_{2}, m_{2}} c_{i_{1}^{\prime}} c_{k_{1}^{\prime}, m_{1}^{\prime}} c_{i_{2}^{\prime}} c_{k_{2}^{\prime}, m_{2}^{\prime}} M\left(i_{1}, k_{1}, i_{2}, k_{2}, i_{1}^{\prime}, k_{1}^{\prime}, i_{2}^{\prime}, k_{2}^{\prime}\right)\right| \\
\leq & {\left[\sum_{i_{1}>b_{n} / 4}+\sum_{k_{1}>b_{n} / 4}+\sum_{i_{2}>b_{n} / 4}+\sum_{k_{2}>b_{n} / 4}+\sum_{i_{1}^{\prime}>b_{n} / 4}+\sum_{k_{1}^{\prime}>b_{n} / 4}+\sum_{i_{2}^{\prime}>b_{n} / 4}+\sum_{k_{2}^{\prime}>b_{n} / 4}\right]\left|c_{i_{1}} c_{k_{1}, m_{1}} c_{i_{2}} c_{k_{2}, m_{2}} c_{i_{1}^{\prime}} c_{k_{1}^{\prime}, m_{1}^{\prime}} c_{i_{2}^{\prime}} c_{k_{2}^{\prime}, m_{2}^{\prime}} M\left(i_{1}, k_{1}, i_{2}, k_{2}, i_{1}^{\prime}, k_{1}^{\prime}, i_{2}^{\prime}, k_{2}^{\prime}\right)\right| } \\
= & \sum_{i=1}^{9} g_{i},
\end{aligned}
$$

where $M\left(i_{1}, k_{1}, i_{2}, k_{2}, i_{1}^{\prime}, k_{1}^{\prime}, i_{2}^{\prime}, k_{2}^{\prime}\right)=\operatorname{cov}\left(y_{t_{1}-i_{1}} y_{t_{1}-k_{1}} y_{t_{2}-i_{2}} y_{t_{2}-k_{2}}, y_{t_{1}^{\prime}-i_{1}^{\prime}} y_{t_{1}^{\prime}-k_{1}^{\prime}} y_{t_{2}^{\prime}-i_{2}^{\prime}} y_{t_{2}^{\prime}-k_{2}^{\prime}}\right)$. By Cauchy-Schwarz inequality, we can show that

$$
\begin{aligned}
\left|M\left(i_{1}, k_{1}, i_{2}, k_{2}, i_{1}^{\prime}, k_{1}^{\prime}, i_{2}^{\prime}, k_{2}^{\prime}\right)\right| & \leq \sqrt{E\left(y_{t_{1}-i_{1}} y_{t_{1}-k_{1}} y_{t_{2}-i_{2}} y_{t_{2}-k_{2}}\right)^{2} E\left(y_{t_{1}^{\prime}-i_{1}^{\prime}} y_{t_{1}^{\prime}-k_{1}^{\prime}} y_{t_{2}^{\prime}-i_{2}^{\prime}} y_{t_{2}^{\prime}-k_{2}^{\prime}}\right)^{2}} \\
& \leq E y_{t}^{8}<\infty .
\end{aligned}
$$

Since $c_{i}=O\left(\rho^{i}\right)$ and $c_{i, m}=O\left(\rho^{i}\right)$ for some $\rho \in(0,1)$, it is straightforward to see that

$$
g_{i} \leq C \rho^{b_{n} / 4}, \text { for } 1 \leq i \leq 8 .
$$

Furthermore, the Davydov's inequality in Davydov (1968) implies that

$$
\begin{aligned}
g_{9} \leq & C \sum_{i_{1}, k_{1}, i_{2}, k_{2}, i_{1}^{\prime}, k_{1}^{\prime}, i_{2}^{\prime}, k_{2}^{\prime} \leq b_{n} / 4}\left\|y_{t_{1}-i_{1}} y_{t_{1}-k_{1}} y_{t_{2}-i_{2}} y_{t_{2}-k_{2}}\right\|_{2+\nu}\left\|y_{t_{1}^{\prime}-i_{1}^{\prime}} y_{t_{1}^{\prime}-k_{1}^{\prime}} y_{t_{2}^{\prime}-i_{2}^{\prime}} y_{t_{2}^{\prime}-k_{2}^{\prime}}\right\|_{2+\nu} \\
& \times\left[\alpha_{y}\left(\left\lfloor\frac{b_{n}}{2}\right\rfloor\right)\right]^{\nu /(2+\nu)}\left|c_{i_{1}} c_{k_{1}, m_{1}} c_{i_{2}} c_{k_{2}, m_{2}} c_{i_{1}^{\prime}} c_{k_{1}^{\prime}, m_{1}^{\prime}} c_{i_{2}^{\prime}} c_{k_{2}^{\prime}, m_{2}^{\prime}}\right| \\
\leq & C\left(E y_{t}^{8+4 \nu}\right)\left[\alpha_{y}\left(\left\lfloor\frac{b_{n}}{2}\right\rfloor\right)\right]^{\nu /(2+\nu)} \\
& \times \sum_{i_{1}, k_{1}, i_{2}, k_{2}, i_{1}^{\prime}, k_{1}^{\prime}, i_{2}^{\prime}, k_{2}^{\prime} \leq b_{n} / 4}\left|c_{i_{1}} c_{k_{1}, m_{1}} c_{i_{2}} c_{k_{2}, m_{2}} c_{i_{1}^{\prime}} c_{k_{1}^{\prime}, m_{1}^{\prime}} c_{i_{2}^{\prime}} c_{k_{2}^{\prime}, m_{2}^{\prime}}\right| \\
\leq & C\left[\alpha_{y}\left(\left\lfloor\frac{b_{n}}{2}\right\rfloor\right)\right]^{\nu /(2+\nu)} .
\end{aligned}
$$

Therefore, since $\lim _{k \rightarrow \infty} k^{2}\left[\alpha_{y}(k)\right]^{\nu /(2+\nu)}=0$, it follows that

$$
\frac{1}{n^{2}} \sum_{s=1}^{L_{n}} \sum_{\left|s^{\prime}-s\right|>1} z\left(s, s^{\prime}\right) \leq O\left(\frac{L_{n}^{2} b_{n}^{4}}{n^{2}}\right)\left[\rho^{b_{n} / 4}+\left[\alpha_{y}\left(\left\lfloor\frac{b_{n}}{2}\right\rfloor\right)\right]^{\nu /(2+\nu)}\right]=o(1) .
$$

By (A41)-(A43), we know that (i) holds. 
For (ii), since $E\left(w_{t}^{*}\right)^{4}<\infty$, by Holder's inequality and the fact that $b_{n}=o\left(n^{1 / 3}\right)$, we have

$$
\begin{aligned}
\sum_{s=1}^{L_{n}} E\left\{E^{*}\left[\left|H_{s n}^{*}\right|^{2} I\left(\left|H_{s n}^{*}\right|>\varepsilon\right)\right]\right\} & \leq C \sum_{s=1}^{L_{n}} E\left(E^{*}\left|H_{s n}^{*}\right|^{4}\right) \\
& =O\left(\frac{1}{n^{2}}\right) \sum_{s=1}^{L_{n}} E\left\{\sum_{t \in B_{s}} \sum_{j=1}^{K}\left[e_{t, j}-E\left(e_{t, j}\right)\right]\right\}^{4} \\
& =O\left(\frac{L_{n} b_{n}^{4}}{n^{2}}\right)=o(1),
\end{aligned}
$$

i.e., (ii) holds. This completes the proof of claim (d).

Q.E.D.

[1] Box, G.E.P. \& PIERCE, D.A. (1970). Distribution of the residual autocorrelations in autoregressive integrated moving average time series models. Journal of the American Statistical Association 65, 1509-1526.

[2] BRockwell, P.J. \& Davis, R.A. (1991). Time Series: Theory and Methods. (2nd ed.). Springer-Verlag, New York.

[3] BRÜGgemann, R., Jentsch, C. \& TRenkler, C. (2014). Inference in VARs with conditional heteroskedasticity of unknown form. Working paper. University of Mannheim.

[4] CARrasco, M. \& Chen, X. (2002). Mixing and moment properties of various GARCH and stochastic volatility models. Econometric Theory 18, 17-39.

[5] Chen, K., Ying, Z., Zhang, H. \& ZhaO, L. (2008). Analysis of least absolute deviation. Biometrika 95, $107-122$.

[6] Chen, K., Guo, S., Lin, Y. \& YIng, Z. (2010). Least absolute relative error estimation. Journal of the American Statistical Association 105, 1104-1112.

[7] Chen, S., Min, W. \& Chen, R. (2013). Model identification for time series with dependent innovations. Statistica Sinica 23, 873-899.

[8] DAVYDOV, Y.A. (1968). Convergence of distributions generated by stationary stochastic processes. Theory of Probability \& Its Applications 13, 691-696.

[9] Delgado, M.A., Hidalgo, J. \& Velasco, C. (2005). Distribution free goodness-of-fit tests for linear processes. Annals of Statistics 33, 2568-2609.

[10] Delgado, M.A. \& Velasco, C. (2011). An asymptotically pivotal transform of the residuals sample autocorrelations with application to model checking. Journal of the American Statistical Association 106, 946-958.

[11] DEO, R.S. (2000). Spectral tests of the martingale hypothesis under conditional heteroskedasticity. Journal of Econometrics 99, 291-315.

[12] Doukhan, P. \& LEÓN, J.R. (1989). Cumulants for stationary mixing random equences and applications to empirical spectral density. Probability and Mathematical Statistics 10, 11-26.

[13] DuRlauf, S.N. (1991). Spectral-based testing of the martingale hypothesis. Journal of Econometrics 50, 355-376.

[14] EsCAnciano, J.C. (2006). Goodness-of-fit tests for linear and non-linear time series models. Journal of the American Statistical Association 101, 531-541.

[15] EsCAnCiAno, J.C. (2007). Model checks using residual marked emprirical processes. Statistica Sinica 17, $115-138$.

[16] Escanciano, J.C. and Lobato, I.N. (2009). An automatic Portmanteau test for serial correlation. Journal of Econometrics 151, 140-149.

[17] Escanciano, J.C., Lobato, I.N. \& ZhU, L. (2013). Automatic specification testing for vector autoregressions and multivariate nonlinear time series models. Journal of Business \& Economic Statistics 31, 426-437.

[18] EsCANCIANO, J.C. \& VELASCO, C. (2006). Generalized spectral tests for the martingale difference hypothesis. Journal of Econometrics 134, 151-185

[19] Eubank, R.L. \& LaRiccia, V.N. (1992). Asymptotic comparison of Cramer-von Mises and nonparametric function estimation techniques for testing goodness-of-fit. Annals of Statistics 20, 2071-2086.

[20] FAN, J. \& JIANG, J. (2007). Nonpatamteric inference with generalized likelihood ratio tests (with discussion). Test 16, 409-478.

[21] FAN, J. \& ZHANG, W. (2004). Generalised likelihood ratio tests for spectral density. Biometrika 91, 195-209.

[22] FrancQ, C., RoY, R. \& ZAKoÏAN, J.M. (2005). Diagnostic checking in ARMA models with uncorrelated errors. Journal of the American Statistical Association 100, 532-544. 
[23] FrancQ, C. \& ZAKOÏAN, J.M. (1998). Estimating linear representations of nonlinear processes. Journal of Statistical Planning and Inference 68, 145-165.

[24] FrancQ, C. \& ZAKoÏAN, J.M. (2010). GARCH Models: Structure, Statistical Inference and Financial Applications. Wiley, Chichester, UK.

[25] FRANSES, P.H. \& VAN DIJK, R. (1996). Forecasting stock market volatility using (non-linear) Garch models. Journal of Forecasting 15, 229-235.

[26] Ghosh, B.K. \& HuAng, W.-M. (1991). The power and optimal kernel of the Bickel-Rosenblatt test for goodness of fit. Annals of Statistics 19, 999-1009.

[27] Granger, C.W.J. \& Anderson, A.P. (1978). An introduction to Bilinear Time Series Models. Vandenhoek and Ruprecht, Gottinger.

[28] Hall, P., Horowitz, J.L. \& Jing, B.-Y. (1995). On blocking rules for the bootstrap with dependent data Biometrica 82, 561-574.

[29] Hamilton, J.D. (1994). Time Series Analysis. Princeton University Press.

[30] Hong, Y. (1996). Consistent testing for serial correlation of unknown form. Econometrica 64, 837-864.

[31] Horowitz, J.L., Lobato, I.N., NAnkervis, J.C. \& SaVin, N.E. (2006). Bootstrapping the Box-Pierce Q test: A robust test of uncorrelatedness. Journal of Econometrics 133, 841-862.

[32] Jentsch, C., Politis, D.N. \& Paparoditis, E. (2014). Block bootstrap theory for multivariate integrated and cointegrated processes. Forthcoming in Journal of Time Series Analysis.

[33] JIN, Z., YING, Z. \& WEI, L.J. (2001). A simple resampling method by perturbing the minimand. Biometrika 88, 381-390.

[34] LING, S. (2007). Self-weighted and local quasi-maximum likelihood estimators for ARMA-GARCH/IGARCH models. Journal of Econometrics 140, 849-873.

[35] Ling, S. \& MCAlEER, M. (2003). Asymptotic theory for a new vector ARMA-GARCH model. Econometric Theory 19, 280-310.

[36] LiU, R.Y. (1988). Bootstrap procedure under some non-i.i.d. models. Annals of Statistics 16, 1696-1708.

[37] LJung, G.M. \& BoX, G.E.P. (1978). On a measure of lack of fit in time series models. Biometrika $\mathbf{6 5}$ 297-303.

[38] Lobato, I.N. (2001). Testing that a dependent process is uncorrelated. Journal of the American Statistical Association 96, 1066-1076.

[39] Paparoditis, E. (2000). Spectral density based goodness-of-fit tests in time series models. Scandinavian Journal of Statistics 27, 143-176.

[40] Paparoditis, E. (2001). On some power properties of goodness of fit tests in time series, in Probability and Statistical Models with Applications, Ed. by Charalambides/Koutras/Balakrishnan, Chapman and Hall, p.333348.

[41] Paparoditis, E. \& Politis, D.N. (2003). Residual-based block bootstrap for unit root testing. Econometrica 71, 813-855.

[42] Parker, C., Paparoditis, E. \& Politis, D.N. (2006). Unit root testing via the stationary bootstrap. Journal of Econometrics 133, 601-638.

[43] Parthas arathy, K.R. (1967). Probability measures on metric spaces. Academic Press. New York.

[44] PHAM, D.T. (1986). The mixing property of bilinear and generalized random coefficient autoregressive models. Stochastic Processes and Their Applications 23, 291-300.

[45] Politis, D.N. \& Romano, J. (1994). Limit theorems for weakly dependent Hilbert space valued random variables with application to the stationary boostrap. Statistica Sinica 4, 461-476.

[46] Romano, J.L. \& Thombs, L.A. (1996). Inference for autocorrelations under weak assumptions. Journal of the American Statistical Association 91, 590-600.

[47] Rosenblatt, M. (1975). A quadratic measure of deviation of two dimensional density estimates and a test of independence. Annals of Statistics 3, 1-14.

[48] Rosenblatt, M. (1985). Stationary Sequences and Random Fields. Birkhäuser, Boston.

[49] Politis, D.N., Romano, J. \& Wolf, M. (1999). Subsampling. Springer-Verla, New York.

[50] SHAO, X. (2011a). A bootstrap-assisted spectral test of white noise under unknown dependence. Journal of Econometrics 162, 213-224.

[51] Shao, X. (2011b). Testing for white noise under unknown dependence and its applications to diagnostic checking for time series models. Econometric Theory 27, 312-343.

[52] Shao, X. \& WU, W.B. (2007). Asymptotic spectral theory for nonlinear time series. Annals of Statistics 35 $1773-1801$.

[53] TSAY, R.S. (2005). Analysis of financial time series (2nd ed.). New York: John Wiley \& Sons, Incorporated.

[54] TSAY, R.S. \& TIAO, G.C. (1984). Consistent estimates of autoregressive parameters and extended sample autocorrelation function for stationary and nonstationary ARMA models. Journal of the American Statistical Association 79, 84-96.

[55] WU, C.F.J. (1986). Jacknife, bootstrap and other resampling methods in regression analysis (with discussion) Annals of Statistics 14, 1261-1350. 
[56] Wu, W.B. (2005). Nonlinear system theory: another look at dependence. Proceedings of National Academy of Science, USA 102, 14150-14154.

[57] Wu, W.B. \& SHAO, X. (2004). Limit theorems for iterated random functions. Journal of Applied Probability 41, 425-436.

[58] ZHU, K. \& LING, S. (2012). The global weighted LAD estimators for finite/infinite variance $\operatorname{ARMA}(p, q)$ models. Econometric Theory 28, 1065-1086. 